\title{
ACCESO A LA EDUCACIÓN UNIVERSITARIA Y GRATUIDAD: MOVILIDAD EDUCACIONAL Y MOVILIDAD TERRITORIAL EN UNA REGIÓN NO METROPOLITANA DE CHILE ${ }^{12}$
}

\author{
Claudio Pareja Pineda ${ }^{3}$; Oscar Mac-Clure ${ }^{4}$, Cristina Pérez \\ Vásquez ${ }^{5}$
}

RESUMEN

Luego de intensos procesos de movilización estudiantil, en 2016 se creó el mecanismo de financiamiento conocido como "gratuidad" para cursar estudios superiores en Chile. Se presume que este mecanismo focaliza la ayuda estatal y promueve que grupos usualmente excluidos ingresen a las universidades, pero existe evidencia controversial al respecto. Utilizando datos administrativos de estudiantes originarios de la Región de los Lagos, se compara el universo que ingresó a la universidad en 2018 con el subgrupo que adicionalmente accedió a "gratuidad". Se examina la asociación entre características sociales de origen y el acceso a la universidad, tanto desde la perspectiva de la movilidad educacional como de la movilidad territorial. Los resultados muestran que los estudiantes de menor estatus socioeconómico acceden principalmente a universidades en la región donde residen. Se concluye que la gratuidad estaría bien focalizada, aunque por sí sola no remueve completamente las barreras para una mayor equidad socioeconómica y territorial.

Conceptos clave: inequidad social, educación superior, política educacional, inequidad espacial, movilidad territorial, segregación residencial, ciudades chilenas, formas de movilidad, aspiraciones.

UNIVERSITY ENROLLMENT AND GRATUIDAD (TUITION-FREE PROGRAM): EDUCATIONAL MOBILITY AND TERRITORIAL MOBILITY IN A NON-METROPOLITAN REGION OF CHILE

\section{ABSTRACT:}

After intense student mobilization processes, in 2016 the Chilean State created the funding mechanism known as gratuidad (tuition-free program) to pursue higher education. It is supposed that this mechanism rationalizes State aid and encourages usually excluded groups to enroll at

1 De manera especial, se agradece el apoyo financiero otorgado por la Universidad de los Lagos para esta investigación.

2 El equipo de la investigación agradece al Departamento de Evaluación, Medición y Registro Educacional (DEMRE) de la Universidad de Chile, por facilitar las bases de datos del Proceso de Admisión a la Educación Superior Universitaria vía Prueba de Selección Universitaria para el desarrollo de esta investigación. También agradece a los funcionarios del Ministerio de Educación, quienes atendieron nuestros requerimientos a través del sistema de transparencia

3 Universidad de Los Lagos, Osorno, Chile. Contacto: claudio.pareja@ulagos.cl

4 Universidad de Los Lagos, Osorno, Chile. Contacto: oscar.macclure@ulagos.cl

5 Universidad de Los Lagos, Osorno, Chile. Contacto: silvia.perez@ulagos.cl 
42 ACCESO A LA EDUCACIÓN UNIVERSITARIA Y GRATUIDAD: MOVILIDAD EDUCACIONAL Y MOVILIDAD TERRITORIAL EN UNA REGIÓN NO METROPOLITANA DE CHILE - C. Pareja Pineda, O. Mac-Clure, C. Pérez Vásquez

universities, but the evidence is controversial. Using administrative data from the Region de Los Lagos students, the universe that entered the university in 2018 is compared to the subgroup that additionally accessed to gratuidad. The association between social characteristics of origin and access to the university is examined, both from the perspective of educational mobility and territorial mobility. The results show that students from lower socioeconomic status access to universities mainly in the region where they reside. It is concluded that the tuition-free program is well focused, although, by itself, it does not remove all the barriers to greater socioeconomic and territorial equity.

Key concepts: social inequality, higher education, educational policy, spatial inequality, territorial mobility, residential segregation, Chilean cities, forms of mobility, aspirations. 


\section{Introducción}

Como efecto de los diversos movimientos estudiantiles posteriores al año 2000 en Chile, especialmente el de 2011, se implementó en 2016 un mecanismo de financiamiento para estudios superiores denominado "gratuidad". Consiste, en términos generales, en un subsidio a la demanda que paga, con recursos públicos, los estudios de estudiantes de menores recursos económicos. Este subsidio solo está disponible para el $60 \%$ más pobre que ingresa a universidades estatales y a algunas privadas que cumplen requisitos acordes con los fines buscados. Se presume que la gratuidad, en tanto nuevo mecanismo de financiamiento tipo voucher, focaliza la ayuda estatal y promueve que grupos usualmente excluidos de la educación superior ingresen a las universidades. Esto es lo que buscamos revisar en este trabajo, específicamente en el caso de una región no metropolitana de Chile y distante del centro del país: la Región de Los Lagos.

En términos generales, con la gratuidad se busca una movilidad educacional más equitativa que en última instancia, contribuya a la movilidad social. Intensos procesos de movilidad social han tenido lugar en diversos países del mundo durante las últimas décadas, desplazando en general hacia arriba a las personas respecto de su posición de origen. En Chile, a pesar de la aguda inequidad de ingresos existente, habría una fluida movilidad social (Torche, 2005), pero existiría una marcada persistencia en cuanto a permanecer en la parte inferior y superior de las clases sociales (Espinoza y Núñez, 2014; OECD, 2018).

La movilidad educacional asociada a los estudios universitarios, en especial cuando se trata de una movilidad educacional intergeneracional, tiene potenciales efectos redistributivos, contribuyendo a la movilidad de ingresos intergeneracional y a reducir las disparidades de ingresos entre grupos particulares (Santiago, Tremblay, Basri \& Arnal, 2008). Se ha sostenido que la educación es actualmente la mayor fuente de movilidad social en Chile, en lo cual sería central la educación universitaria (Torche, 2014). Esto plantea una discusión sobre si los estudiantes de los estratos sociales con menos privilegios están accediendo a dicha 
44 ACCESO A LA EDUCACIÓN UNIVERSITARIA Y GRATUIDAD: MOVILIDAD EDUCACIONAL Y MOVILIDAD TERRITORIAL EN UNA REGIÓN NO METROPOLITANA DE CHILE - C. Pareja Pineda, O. Mac-Clure, C. Pérez Vásquez

educación y si con esto se genera una movilidad educacional. De este modo, una primera pregunta es ¿qué características socioeconómicas tienen quienes, provenientes de una región como la de Los Lagos, se matriculan en universidades, y qué características tiene en particular el subgrupo que accede a la gratuidad para sus estudios?

En países latinoamericanos se han impulsado nuevas políticas sobre el acceso a la universidad. En el caso de Brasil, por ejemplo, durante las últimas décadas se expandió el acceso a la universidad para quienes antes estaban excluidos por sus condiciones socioeconómicas y de raza (Carvalhaes \& Ribeiro, 2019; Filho Crepalde \& Silvera Souza, 2016; Marteleto, Marschner $\&$ Carvalhaes, 2016). Las reformas en Brasil ampliaron el acceso a las universidades públicas a través de sistemas de cuotas basados en desigualdades socioeconómicas y raciales, además de becas y beneficios tributarios. Se ha analizado empíricamente el efecto de las reformas en la reducción de las desventajas de acceso relacionadas con ser no blanco y pobre, concluyendo que, a pesar de los avances, persisten dificultades para los menos privilegiados (Marteleto et al., 2016). También se han investigado desigualdades horizontales en las instituciones universitarias y su efecto en el desempeño universitario de los estudiantes. Se observa que, dentro de una misma carrera, se tiende a equiparar las desigualdades de ingreso, raza y género, pero quienes tienen un origen más aventajado tienen más oportunidades de acceso y un mejor desempeño en carreras más rentables en el mercado y en instituciones universitarias más prestigiadas (Carvalhaes \& Ribeiro, 2019; Filho Crepalde \& Silvera Souza, 2016).

En Chile se ha observado que la promesa del ascenso social mediante los estudios universitarios está lejos de ser una realidad, pues los estudiantes más privilegiados que acceden a universidades de elite son quienes posteriormente obtienen mayores retornos económicos en el mercado laboral (Barozet, Contreras, Espinoza, Gayo y Méndez, 2021). Esto nos plantea una segunda pregunta, acerca de dónde realizan sus estudios los estudiantes de estratos sociales menos privilegiados, en especial quienes acceden a la gratuidad. 
Para estudiar la gratuidad desde la perspectiva de la movilidad educacional, en esta investigación se caracterizará a los estudiantes de la Región de Los Lagos que ingresaron a estudios universitarios, diferenciando si se adjudican o no gratuidad en sus estudios. Se analiza sus posibilidades de movilidad educacional en términos de su transición socioeducacional desde la educación media, considerando el establecimiento de educación secundaria del que provienen. Además, desde un punto de vista intergeneracional, se analiza el nivel de educación formal de los padres. Esto último permite una aproximación a la movilidad educacional intergeneracional, entendida como el "porcentaje de adultos que alcanza un nivel educativo [formal] más alto que sus padres" (OECD, 2018, p. 246).

También se analiza la universidad de destino según tipo de universidad. Se analizan con especial atención aspectos territoriales, como el lugar geográfico de domicilio durante la educación secundaria y el lugar donde el estudiante ingresa a realizar estudios universitarios. Esto permite caracterizar la movilidad territorial, constituida en un componente central de la configuración geográfica y social del país, lo cual requiere considerar tanto la movilidad interregional como la intrarregional (Hidalgo, De Mattos y Arenas, 2009). Por esta razón, sostenemos que la movilidad educacional debe estudiarse conjuntamente con la movilidad territorial. Abarcar ambos aspectos puede contribuir a ampliar el conocimiento sobre un acceso más igualitario a la universidad como una vía para una mayor equidad de oportunidades.

Esta investigación constituye uno de los primeros análisis evaluativos de la gratuidad y se orienta a abordar la interrogante de si este mecanismo de financiamiento para estudios universitarios se focaliza en los menos privilegiados en comparación con el resto de los estudiantes. Son frecuentes los estudios sobre educación a nivel macro o nacional, lo que este trabajo extiende hacia un nivel subnacional, enfocando el caso de una región no metropolitana y distante del centro, la Región de Los Lagos.

De este modo, el objetivo del estudio consiste en analizar las características socioeconómicas y educacionales de los estudiantes de 
46 ACCESO A LA EDUCACIÓN UNIVERSITARIA Y GRATUIDAD: MOVILIDAD EDUCACIONAL Y MOVILIDAD TERRITORIAL EN UNA REGIÓN NO METROPOLITANA DE CHILE - C. Pareja Pineda, O. Mac-Clure, C. Pérez Vásquez

la Región de Los Lagos ingresados con gratuidad a las universidades, en comparación con el total de matriculados procedentes de la misma región. Específicamente, el análisis se focaliza en quienes iniciaron su primer año en carreras universitarias (PSU) durante 2018, examinando aspectos relevantes acerca de su condición socioeconómica de origen y del destino de sus estudios universitarios, y asignando particular atención a la movilidad territorial, es decir, sus traslados por motivos de estudio a nivel intrarregional e interregional.

En una primera parte se revisa la literatura sobre movilidad educacional, así como el vínculo con los estudios universitarios en Chile y otros países. Enseguida, se presentan antecedentes sobre el caso de la Región de Los Lagos, en la cual se focaliza el estudio. En la siguiente parte se expone la metodología del trabajo, incluyendo el diseño del estudio, las variables utilizadas y los métodos estadísticos aplicados. En la sección sobre resultados, se entrega una visión general de la gratuidad en la Región de Los Lagos, así como una visión específica sobre la movilidad que realizan los estudiantes de la región y el tipo de institución en la que se matriculan. Finalmente, se presentan las principales conclusiones de este estudio.

\section{Oportunidad de movilidad educacional: el acceso a la universidad}

2.1. ¿Quién estudia? Acceso a la educación universitaria en Chile

La movilidad educacional intergeneracional, específicamente a través de oportunidades más igualitarias en el acceso a la educación universitaria, en principio podría contribuir a una mayor equidad social.

La ampliación de la cobertura de la educación universitaria en Chile ha sido examinada en diversos estudios (Castillo y Cabezas, 2010; Espinoza, González-Fiegehen y Granda, 2019). Este aumento de cobertura se da en paralelo con la composición estatal/privada del gasto en educación terciaria en Chile, abarcando un 32\% pública y un $68 \%$ privada al año 2016. Esta situación es inversa al promedio OCDE, de $68 \%$ público y $32 \%$ privado, al mismo tiempo que el 
gasto total anual por estudiante terciario en Chile es prácticamente la mitad que el promedio OCDE (Brunner y Labraña, 2018). Chile tuvo en 2013 un gasto en educación superior con relación al PIB que ascendió al 2,4\%, del cual un 0,7\% fue absorbido por el Estado y un 1,7\% fue aportado por el sector privado (Espinoza y González, 2015).

La cobertura universitaria ha aumentado, pero no lo suficiente desde un punto de vista de las desigualdades socioeconómicas. En general, los jóvenes de sectores populares estudian menos años y entran a trabajar a edades más tempranas que los de clase alta (Dávila y Ghiardo, 2005, citado en López, Mella y Cáceres, 2018). Si el quintil más pobre cuadruplicó su participación desde un 4,4\% en 1990 a 17,3\% en 2006, el más rico lo duplicó de un 40,7\% a 80,0\% (Espinoza y González, 2013). Más aún, aunque los estudiantes de escuelas privadas llegan solo al 8\% de la matrícula en la educación secundaria (2005/2006), éstos alcanzan un 22\% de la matrícula en universidades del Consejo de Rectores de Universidades Chilenas (CRUCH) (Espinoza y González, 2013). Adicionalmente, existen grupos de la sociedad cuyo acceso a la universidad ha sido dificultoso, no solamente por falta de ingresos, como ocurre con quienes pertenecen a pueblos indígenas (Abarca y Zapata, 2005).

En Chile, el ingreso a las universidades públicas y a las privadas de mayor prestigio ha tenido como antecedente la Prueba de Selección Universitaria ${ }^{6}$ (PSU). Esta prueba consiste en un conjunto de dos subpruebas obligatorias, Lenguaje y Matemáticas, y dos optativas, Historia y Ciencias [naturales]. La literatura ha reportado que, en general, los resultados en la PSU correlacionan positivamente con el nivel socioeconómico del estudiante (González, Arancibia y Boyanova, 2017).

La PSU emerge como elemento crucial en el acceso a la universidad, encontrándose una asociación positiva entre los puntajes en las pruebas de selección universitaria y las tasas de postulación al sistema de admisión (Canales, 2016; Valdivieso, Antivilo y Barrios,

6 El Ministerio de Educación, en marzo de 2020, estableció que el sistema de admisión para las universidades fuera modificado, implementando para los ingresos 2021 y 2022 un nuevo instrumento llamado "Prueba de Transición Universitaria". 
48 ACCESO A LA EDUCACIÓN UNIVERSITARIA Y GRATUIDAD: MOVILIDAD EDUCACIONAL Y MOVILIDAD TERRITORIAL EN UNA REGIÓN NO METROPOLITANA DE CHILE - C. Pareja Pineda, O. Mac-Clure, C. Pérez Vásquez

2006). Analizando posibles motivos por los cuales estudiantes de sectores vulnerables no logran ser representados en la educación superior, algunos autores refieren barreras de tipo económicofinancieras (Espinoza y González, 2013), mientras que otros relevan aquello que los propios jóvenes destacan como importante: puntaje obtenido en la PSU, costo o arancel de la carrera y promedio de notas alcanzado en la enseñanza media (Castillo y Cabezas, 2010; Sepúlveda y Valdebenito, 2014). Asimismo, los puntajes PSU crecen a medida que aumenta el nivel educacional de ambos padres del postulante, por lo cual tienen mayores probabilidades de postular al sistema los estudiantes con padres universitarios y/o en ocupaciones profesionales, en comparación con aquellos cuyos padres no tienen educación universitaria y se desempeñan en ocupaciones no profesionales (Canales, 2016).

\section{2. ¿Dónde estudiar? Segregación de la oferta de educación universitaria en Chile y movilidad territorial}

Una vez decidido postular, las diferencias socioeconómicas continúan enseguida afectando dónde postular. Según refieren Jarpa-Arriagada y Rodríguez-Garcés (2018), estudiantes de primera generación en la universidad eligen carreras de baja selectividad —en las que un $50 \%$ de la matrícula histórica no supera los 500 puntos PSU-y universidades regionales, privilegiando la cercanía territorial. Esto último, señalan los autores, reduce los costos y además brinda la posibilidad de mantener redes de apoyo necesarias cuando se aventuran por primera vez en el espacio universitario. Además, ajustan sus elecciones de carrera atendiendo disponibilidad de becas, ayudas estudiantiles o cupos de equidad (Jarpa-Arriagada y Rodríguez-Garcés, 2018). A esto se agrega las expectativas académicas: la inseguridad frente a las exigencias académicas en la educación superior incide de modo relevante en las orientaciones de los estudiantes de menores recursos (Castillo y Cabezas, 2010; Sepúlveda y Valdebenito, 2014).

Este desigual ingreso al sistema universitario, desde la perspectiva del destino de los estudiantes, tiene como correlato una estratificación de las instituciones, configurando un sistema de 
universidades que, aunque iguales en teoría, reciben distinto tipo de estudiantes. Muñoz y Blanco (2013) presentan una clasificación de universidades en cinco grupos, según variables como selectividad en el ingreso, tamaño y productividad investigativa. Los cinco grupos son universidades "[de] investigación", "masivas", "de acreditación", "elitistas" y "no elitistas". Las universidades de acreditación serían aquellas acreditadas y con una actividad importante de investigación, aunque no tanta como las universidades de investigación. Las universidades no elitistas corresponden a universidades pequeñas, con foco en el pregrado y con bajos niveles de selectividad de estudiantes. Mientras que la Universidad de Chile, la Pontificia Universidad Católica de Chile y la Universidad de Concepción se encuentran en la categoría de investigación, el resto de las universidades $\mathrm{CRUCH}$ están en la categoría "de acreditación" o la de "no elitistas".

De un modo general, la promesa de la movilidad educacional intergeneracional enfrenta dificultades en diversos países. A este respecto, resulta de interés el estudio de Reay (2018) acerca del ingreso a universidades de elite por parte de estudiantes de clase trabajadora en Gran Bretaña. Estos estudiantes enfrentan no solo la inseguridad de lograr elevadas metas académicas sino también la de ser aceptados en un campo en el que estudiantes de mayor estatus poseen capital cultural, social y económico. Al carecer de esos capitales en términos relativos, los estudiantes menos privilegiados se perciben a sí mismos como marginalizados y lejos del discurso sobre la movilidad educacional, por lo cual es posible que esa expectativa opere como un factor de autoexclusión respecto del acceso a esas universidades de elite (Loveday, 2014; Reay, 2018). En un estudio similar realizado en el contexto colombiano, estudiantes becados muestran temor a no lograr los resultados esperados, por lo que una emoción bastante común es la ansiedad académica relativa al miedo de no concretar la deseada movilidad social (Álvarez Rivadulla, 2019). Se releva la importancia de estudiar la complejidad del mundo subjetivo de estudiantes pobres que ingresan a universidades de elite, para comprender las barreras, pero también los beneficios que este tipo de iniciativas conlleva, por ejemplo, al derribar algunos prejuicios y al mismo tiempo hacer más explícita la desigualdad de origen (Álvarez Rivadulla, 2019). 
50 ACCESO A LA EDUCACIÓN UNIVERSITARIA Y GRATUIDAD: MOVILIDAD EDUCACIONAL Y MOVILIDAD TERRITORIAL EN UNA REGIÓN NO METROPOLITANA DE CHILE - C. Pareja Pineda, O. Mac-Clure, C. Pérez Vásquez

Las desigualdades sociales en el ámbito educacional tienen un correlato en el espacio físico. A pesar de que eso es bien sabido, el vínculo entre el espacio social y el espacio físico es más complejo que una mera relación de espejo (Bourdieu, 2007; Sabatini, Cáceres y Cerda, 2001). El vínculo entre el espacio social y el espacio físico en el ámbito educacional se reflejaría en la distribución geográfica de las universidades, así como en los movimientos que realizan los estudiantes en el territorio geográfico en busca de mejores oportunidades educacionales.

En efecto, los diversos tipos de instituciones universitarias están relacionados con la distribución geográfica de las universidades. Donoso, Arias, Weason y Frites (2012) indican que la expansión de la cobertura y matrícula en educación superior ha sido implementada desde una visión de mercado más que una lógica vinculada con las necesidades del entorno y la coherencia con un proyecto de desarrollo territorial. Esto implica que la oferta de educación terciaria en Chile se distribuye desigualmente entre regiones e intrarregión. Los autores señalan que las regiones con ciudades metropolitanas son claramente líderes en oferta de educación superior, mientras que las demás presentan menor variedad en el tipo de instituciones y en el número de vacantes (Donoso et al., 2012). De este modo, la existencia de una oferta de educación segmentada social, económica y territorialmente puede eventualmente resolver la creciente demanda de acceso a la universidad, sin modificar mayormente las diferencias de origen y segmentación social prevalecientes en la sociedad chilena (Sepúlveda y Valdebenito, 2014).

La conjugación de una segregación según el estatus social de los estudiantes y según el lugar geográfico, abre la posibilidad de que estudiantes se trasladen en busca de una "mejor" universidad. Una investigación analiza en detalle este fenómeno, usando como caso de estudio la Región de Los Lagos y mostrando que los estudiantes con ingresos familiares bajos tienden a estudiar en la misma región (Saravia, 2018a, 2018b). Entre quienes se trasladan fuera de su lugar de origen, es decir, desarrollan una movilidad territorial, muchas veces sus familias deben hacer un esfuerzo económico mayor para solventar los estudios. Asimismo, según la investigación mencionada, los años 
de duración de los estudios universitarios tienen un costo económico para quienes migran e implican también largas experiencias de vida, que varían dependiendo de la evolución de su movilidad territorial.

Lo anterior refuerza la importancia de incorporar un enfoque de movilidad territorial en el estudio del acceso a la universidad. Además de los traslados entre regiones, es importante observar que la movilidad territorial por motivos de estudios universitarios también puede asumir la forma de viajes diarios. En el caso de regiones no metropolitanas, ello sería cada vez más común entre estudiantes de estratos socioeconómicos más bajos, que acceden a la oferta universitaria de ciudades intermedias pero que residen fuera de éstas. Esto implica viajes a esas ciudades, por lo cual las distancias espaciales ejercen efectos en el rendimiento académico y en las formas de experimentar la vida universitaria de esos estudiantes, limitando sus oportunidades de desarrollo académico (Riquelme, 2017).

Al mismo tiempo, las personas de regiones no metropolitanas, en búsqueda de un mejor futuro a través de la educación universitaria, buscan una institución de "prestigio" y un territorio acorde, lo que, en general, corresponde a territorios metropolitanos (Saravia, 2018a). En definitiva, la oferta académica universitaria en Chile está segregada (Muñoz y Blanco, 2013) y esta segregación tiene un correlato a nivel de regiones (Donoso et al., 2012).

Sobre esa base, sostenemos que la movilidad territorial es relevante respecto de las oportunidades para la realización de estudios universitarios. Esto involucra considerar la movilidad desde la comuna de origen a una universidad en la misma región, pero en otra comuna — movilidad intrarregional—, y la movilidad para estudiar en otra región —movilidad interregional—, así como la distancia relativa hacia el lugar de destino. En el caso de la región estudiada, la movilidad intrarregional es frecuente y corresponde en una alta proporción a viajes diarios para la realización de estudios, aunque en una parte menor puede implicar un cambio de residencia a otra comuna de la región, es decir una migración al menos temporal. La movilidad interregional consiste en traslados a regiones distintas de la analizada, que en alta proporción corresponden a migración, aunque 
52 ACCESO A LA EDUCACIÓN UNIVERSITARIA Y GRATUIDAD: MOVILIDAD EDUCACIONAL Y MOVILIDAD TERRITORIAL EN UNA REGIÓN NO METROPOLITANA DE CHILE - C. Pareja Pineda, O. Mac-Clure, C. Pérez Vásquez

en una parte menor involucra viajes diarios a una región cercana. Así, la movilidad territorial engloba el total de ambos tipos de movilidad.

\subsection{Efectos de la ampliación del financiamiento en el acceso universitario}

En los últimos veinte años la educación superior de Chile ha incorporado diversas formas de subsidios institucionales, ya sean "subsidios a la oferta" o "subsidios a la demanda" (Ruff Escobar, Ruiz Toledo, Matheu Pérez y Juica Martínez, 2020). Entre los primeros se encuentra el financiamiento a universidades mediante aportes fiscales directos e indirectos, fondos concursables y fondos por convenios de desempeño en acuerdo con el Estado. Entre los segundos se incluyen becas de arancel, créditos universitarios solidarios o con aval del Estado, todos los cuales exigen que los estudiantes alcancen puntajes PSU mínimos para poder acceder a éstos. Entre estos subsidios a la demanda también se encuentra la gratuidad, siendo el único que no exige puntaje PSU.

Respecto del apoyo financiero para el acceso universitario, Estados Unidos ha sido comparado con Chile, al existir una amplia oferta privada además de la pública, la cual, al igual que en Chile, varía en términos de selectividad y calidad (Delisle \& Bernasconi, 2018). Además, la proporción de gasto público versus gasto privado también es similar (Brunner y Labraña, 2018). Sin embargo, se diferencian ambos países en cuanto a que en Estados Unidos la mayor parte de los estudiantes está matriculado en instituciones públicas (Delisle \& Bernasconi, 2018).

Angrist, Autor, Hudson y Pallais (2016) evalúan los impactos de un programa de becas a través de un diseño experimental longitudinal en el Estado de Nebraska, Estados Unidos. En este programa, después de revisar que se cumplía un mínimo estándar de buen rendimiento y necesidades financieras, parte de las becas se pasaron a entregar de manera aleatoria (Angrist et al., 2016). Los postulantes deben estudiar en universidades y centros de formación técnica (two-year colleges, four-year colleges) públicos. Los beneficios incluyen apoyos financieros por hasta cinco años y la participación en comunidades 
de aprendizaje, cuyo objetivo es potenciar el desarrollo académico y social. La evaluación concluyó que las tasas de acceso y de retención aumentaron, especialmente en grupos con usualmente altas tasas de deserción: estudiantes no blancos, primera generación en estudios universitarios y con bajo rendimiento en la educación secundaria. Así, los autores destacan la paradoja de que ayudas basadas en "méritos" anteriores tenderían a una menor focalización respecto de quienes más podrían beneficiarse de la ayuda financiera.

En referencia a Chile, se ha aportado evidencia en el sentido de que el apoyo financiero para estudios universitarios — como el crédito universitario- beneficia a estudiantes de menores ingresos relativos. Un estudio econométrico concluyó que el acceso al crédito (Fondo Solidario y CAE) duplica la probabilidad de acceder a la universidad para estudiantes justo en el margen del puntaje PSU obligatorio para poder acceder a estos créditos (Solis, 2017). En específico, estudiantes que apenas cumplían el requisito académico para acceder al crédito, es decir, que obtuvieron no más de 477 en la PSU (hasta 2 puntos más que los 475 necesarios), tuvieron una tasa de matrícula del 38\% frente a un 19\% de estudiantes con 473 o más puntos (pero menos de 475). Este estudio además concluyó que la mejora en la probabilidad de acceso al crédito fue superior en estudiantes del quintil de ingresos más bajo, logrando mitigar completamente las diferencias por estrato socioeconómico.

Sin embargo, los estudiantes tienen mejores resultados en términos educacionales y de inserción posterior en el mercado laboral cuando acceden a universidades de mayor calidad (Bucarey, Contreras \& Muñoz, 2020). Considerando que estas universidades se concentran en Santiago y otras grandes ciudades del país, las posibilidades de movilidad territorial para acceder a ellas por parte de quienes viven en lugares más alejados del país es un factor relevante desde el punto de vista del apoyo financiero para estudios universitarios.

Una innovación en el mecanismo de financiamiento gratuidad en Chile es que éste no considera requisitos académicos (Deslisle $\&$ Bernasconi, 2018). Así, los autores sostienen que los estudiantes que se matriculan en instituciones menos selectivas serían aquellos 
54 ACCESO A LA EDUCACIÓN UNIVERSITARIA Y GRATUIDAD: MOVILIDAD EDUCACIONAL Y MOVILIDAD TERRITORIAL EN UNA REGIÓN NO METROPOLITANA DE CHILE - C. Pareja Pineda, O. Mac-Clure, C. Pérez Vásquez

que más se beneficiarían por la gratuidad. Concluyen que, aunque el mismo efecto alcanzado por la omisión de requisitos académicos pudiera obtenerse a través de becas (means-tested grants), el proceso de postulación a becas es menos transparente y entendible, pudiendo llevar a estudiantes a pensar que la educación superior está fuera de su alcance financiero. Más aún, se ha informado que procesos burocráticos de postulación a becas beneficiarían mayormente a estudiantes con la motivación y conocimiento especializado suficientes para realizar la tramitación, quienes usualmente no son quienes más necesitan la ayuda (Goldrick-Rab, Kelchen, Harris \& Benson, 2016).

Complementando esta visión, Bucarey (2018) observa que, con el subsidio directo a la demanda para estudiantes de bajos ingresos en Chile, los estudiantes que ingresan a universidades no selectivas serían los más beneficiados, pero alerta que las universidades selectivas podrían volverse aún más elitistas. Esto se debe a que una reducción general de los costos para el usuario final, a través de un subsidio a la demanda - tipo gratuidad - puede causar que estudiantes de clase media postulen a universidades más caras, las cuales, en general, son más atractivas y selectivas. Asumiendo niveles de matrícula estables, esto puede causar que estudiantes de ingresos más bajos queden fuera de las universidades de más prestigio, pues, en promedio, ellos tienen menores puntajes.

Argumentando acerca de un efecto redistributivo negativo, Brunner y Labraña citan un informe del Estado chileno para indicar que el 89\% de los beneficiados por la gratuidad en 2017 proviene del cuarto y quinto decil (Brunner y Labraña, 2018). Por otro lado, un estudio comparativo de las reformas recientes en Ecuador y Chile concluye que en ambos países ha crecido el porcentaje de estudiantes en quintiles de ingreso bajos que accedieron a la educación, aunque en Chile más que en Ecuador (Espinoza et al., 2019).

Así, la focalización de la gratuidad ha sido reportada de manera contradictoria. Desde el punto de vista del logro de un sistema más igualitario, sus efectos en el acceso a la educación universitaria no son claros. Por esto, consideramos relevante aportar nueva evidencia que contribuya a una mejor comprensión de sus efectos. A partir de 
lo expuesto, se podría sostener que, hipotéticamente: a) la gratuidad favorece principalmente a estudiantes menos privilegiados; b) las características de origen entre los estudiantes adscritos a la gratuidad condicionan la permanencia en la región para estudios universitarios.

\section{Métodos y datos}

\subsection{Diseño general del estudio}

El diseño general de este estudio es cuantitativo, basado inicialmente en métodos de estadística descriptiva. Esto bosqueja relaciones entre variables que habitualmente se consideran independientes y que se identifican como dependientes de acuerdo con lo expuesto: el acceso a la educación universitaria y el lugar de matrícula universitaria.

Sin embargo, debido a que, hasta lo que sabemos, hay un escaso conocimiento sobre el objeto de estudio, el trabajo tiene un carácter más bien exploratorio. Se requiere inicialmente describir los resultados observados para, posteriormente, formular hipótesis más específicas sobre los aspectos de mayor relevancia respecto de los objetivos del estudio.

Desde un punto de vista metodológico, se justifica analizar el caso de estudiantes provenientes de la Región de Los Lagos. Como ocurre en otras regiones del país, esta región está alejada geográficamente del centro del país, pero con una oferta universitaria variada; dicha oferta se concentra principalmente en su capital regional, Puerto Montt, y en menor medida en la ciudad de Osorno. Además, la región a analizar incluye tanto ciudades intermedias como territorios menos urbanizados que la rodean, y cuenta con una importante presencia indígena, compartiendo así características socioeconómicas similares a las de otras regiones de la zona sur del país.

El estudio se circunscribe a estudiantes que ingresaron a primer año de universidad durante 2018 y que se graduaron de establecimientos de educación secundaria regular . Más aún,

7 Se excluyeron egresados de la educación secundaria para adultos. 
considera solo a estudiantes matriculados en carreras universitarias cuyo ingreso requiere puntaje PSU. Cabe notar que esta especificación del destino universitario de los estudiantes se traduce en incluir universidades de todo el país, aunque solamente aquellas que utilizan la PSU como mecanismo de selección para el ingreso.

Observando que son frecuentes los estudios de alcance nacional, este estudio ofrece una doble innovación metodológica. La primera consiste en centrar la muestra de estudiantes en un territorio subnacional específico. La segunda surge al considerar que el destino de estudios universitarios no solo ocurre en instituciones específicas, sino que en territorios delimitados.

\subsection{Variables y bases de datos utilizadas}

De acuerdo con lo expuesto, en este trabajo se compara el universo de estudiantes de la Región de los Lagos con los adscritos a gratuidad, utilizando la cohorte de ingreso 2018 a carreras PSU. Sobre esta base, se utilizan las variables sobre el estudiantado y la institución universitaria que se presentan en la tabla 1.

Tabla 1.

Lista de variables utilizadas en el estudio

\begin{tabular}{lll}
\hline Características del estudiantado & Características de la institución universitaria \\
\hline 1) Sexo & 8) & Universidad según tipo de institución \\
2) Nivel educativo máximo & (Estatal CRUCH; Privada CRUCH; \\
alcanzado por la madre y/o el & Privada) \\
padre & 9) & $\begin{array}{l}\text { Región donde se emplaza la sede de } \\
\text { matrícula universitaria }\end{array}$ \\
3) Comuna de domicilio al & 10) Comuna donde se emplaza la sede de \\
momento de egreso & matrícula universitaria \\
4) Ascendencia étnica declarada & 11) Existencia de movilidad territorial: \\
5) Establecimiento secundario de & permanencia, movilidad intrarregional \\
egreso & o movilidad interregional \\
6) $\begin{array}{l}\text { Dependencia del establecimiento } \\
\text { de egreso (municipal, particular }\end{array}$ & 12) Adscripción del estudiante a la \\
subvencionado, particular & gratuidad \\
pagado) & \\
7) Categoría de vulnerabilidad & \\
escolar del establecimiento de & \\
egreso & \\
\hline
\end{tabular}

Fuente: Elaboración propia. 
Los datos utilizados en el estudio provienen, en primer lugar, de bases de datos solicitadas al DEMRE (Departamento de Evaluación, Medición y Registro Educacional de la Universidad de Chile) a través de su portal para investigadores, el cual contiene toda la información sobre el universo de estudiantes PSU. En segundo lugar, se emplean datos del MINEDUC (Ministerio de Educación) obtenidos a través del sistema de transparencia, que aportan la información respecto del subgrupo de estudiantes que accedieron a la gratuidad.

Desde el punto de vista de los datos utilizados, un aporte original de esta investigación consiste en que ambas fuentes de datos se analizan en forma conjunta, aplicándolas a una región en particular. Sin embargo, la base de datos utilizada para el universo PSU y la empleada para el subgrupo con gratuidad no identifican a cada estudiante, lo cual implica una limitación en cuanto a la viabilidad de identificar dentro de ese universo PSU a quienes adscribieron o no a la gratuidad.

Los datos indicados son complementados con información publicada en los sitios web oficiales del MINEDUC respecto del IVE (Índice de Vulnerabilidad Escolar) de cada establecimiento. En Chile, el sistema educativo escolar secundario considera tres tipos de establecimiento según dependencia: particulares pagados, particulares subvencionados por el Estado y municipales también subvencionados por el Estado. En general, la literatura que estudia el acceso a la educación superior distingue el establecimiento educacional de egreso según esta diferencia en la dependencia (ver, por ejemplo, Espinoza y González, 2015). En este estudio, si bien utilizamos esta categorización por dependencia, esta información se complementa con el uso del IVE, porque permite una clasificación más fina de los establecimientos, al incorporar la heterogeneidad de la matrícula en términos socioeconómicos dentro de cada dependencia.

El IVE es calculado cada año por JUNAEB (Junta Nacional de Auxilio Escolar y Becas) del MINEDUC sobre la base de una encuesta censal realizada en primer año de cada nivel formativo acerca de los ingresos y niveles educativos familiares (Bravo y Verdugo, 2007), y otros antecedentes, como la participación en programas sociales para 
58 ACCESO A LA EDUCACIÓN UNIVERSITARIA Y GRATUIDAD: MOVILIDAD EDUCACIONAL Y MOVILIDAD TERRITORIAL EN UNA REGIÓN NO METROPOLITANA DE CHILE - C. Pareja Pineda, O. Mac-Clure, C. Pérez Vásquez

la superación de la pobreza (MINEDUC, n.d.). El IVE representa con un porcentaje entre 0 y 100 la cantidad de estudiantes que se consideran vulnerables. Así, a mayor IVE, más estudiantes con bajos ingresos y condiciones de pobreza por establecimiento.

En esta investigación se utiliza el IVE para Enseñanza Media como indicador principal del nivel socioeconómico de los estudiantes, por razones que han sido abordadas en la literatura sobre el tema. Por un lado, se ha informado que entre establecimientos subvencionados y municipales se alcanzan niveles similares de efectividad y calidad en la gestión (Castillo, González y Puga, 2011). En este sentido, las escuelas municipales y las subvencionadas, corrigiendo por nivel de ingreso de los estudiantes, alcanzarían niveles similares en las pruebas estandarizadas estatales SIMCE y tendrían niveles similares de recursos para perfeccionamientos de los docentes (Castillo et al., 2011). Por otro lado, una reciente investigación estudia el efecto de un programa de apoyo escolar en los puntajes PSU, controlando por vulnerabilidad del establecimiento y talento personal (González et al., 2017). Para la vulnerabilidad escolar, se clasifica a los establecimientos de egreso según su IVE y no según dependencia. Los autores concluyen que este índice de vulnerabilidad explica, al menos en parte, los resultados en los puntajes PSU de la población estudiada (González et al., 2017).

Para analizar la distribución según IVE, se agrupan establecimientos en quintiles ${ }^{8}$, utilizando la base de datos disponible para establecimientos de educación secundaria. Adicionalmente, se agrega una categoría para incluir establecimientos para los cuales MINEDUC no calcula el IVE, los establecimientos particulares pagados, que son denominados "Sin IVE (particular pagado)". Las categorías de IVE que se utilizan en el estudio y la cantidad de establecimientos en cada una de ellas, desagregada por dependencia, se muestra en la tabla 2. Como se puede apreciar, la agrupación propuesta entrega categorías de establecimientos similares en número y que, además, incorporan la diversidad de establecimientos presentes

8 Corresponde al 20\% de los establecimientos para los cuales se calcula el IVE en cada categoría. Esto no implica, per se, una distribución particular sobre los estudiantes. 
dentro de cada dependencia. A modo de ejemplo, si se observa los establecimientos con mayor vulnerabilidad (Quintil I), se aprecia que en su mayoría son municipales y solo un tercio corresponden a establecimientos particulares subvencionados. La proporción de estos últimos aumenta a medida que ascienden los quintiles de IVE.

Tabla 2.

Cantidad de establecimientos secundarios a nivel nacional según vulnerabilidad (IVE) y dependencia

\begin{tabular}{llcccc}
\hline Clasificación vulnerabilidad (IVE) & Municipal & $\begin{array}{c}\text { Particular } \\
\text { subvencionado } \\
\text { y delegado }\end{array}$ & $\begin{array}{c}\text { Particular } \\
\text { pagado }\end{array}$ & Total n \\
\hline Quintil I & $90.17 \%+$ (Más vulnerables) & $64 \%$ & $36 \%$ & $0 \%$ & 453 \\
Quintil II & $82.53 \%-90.16 \%$ & $53 \%$ & $47 \%$ & $0 \%$ & 476 \\
Quintil III $72.39 \%-82.52 \%$ & $26 \%$ & $73 \%$ & $0 \%$ & 487 \\
Quintil IV & $56.13 \%-72.38 \%$ & $17 \%$ & $82 \%$ & $1 \%$ & 497 \\
Quintil V & $<=56.12 \%$ (menos vulnerables) & $4 \%$ & $88 \%$ & $7 \%$ & 494 \\
Sin IVE & Particular pagado & $0 \%$ & $0 \%$ & $100 \%$ & 425 \\
\hline & Total & 774 & 1.590 & 468 & 2.832 \\
& & $27 \%$ & $56 \%$ & $17 \%$ & $100 \%$ \\
\hline
\end{tabular}

Nota: Se considera establecimientos que tuvieron al menos un estudiante que se matriculó en primer año de una carrera PSU.

Fuente: Elaboración propia, de acuerdo con información entregada por MINEDUC y DEMRE, año de ingreso 2018.

La clasificación de estudiantes según IVE muestra una relativa consistencia con los niveles de ingreso bruto familiar. Esto se aprecia en la tabla 3, en la que se presenta la distribución de niveles de vulnerabilidad según estratos de ingreso, respecto del total de estudiantes de la Región de Los Lagos que ingresaron a la universidad en el año 2018. 
60 ACCESO A LA EDUCACIÓN UNIVERSITARIA Y GRATUIDAD: MOVILIDAD EDUCACIONAL Y MOVILIDAD TERRITORIAL EN UNA REGIÓN NO METROPOLITANA DE CHILE - C. Pareja Pineda, O. Mac-Clure, C. Pérez Vásquez

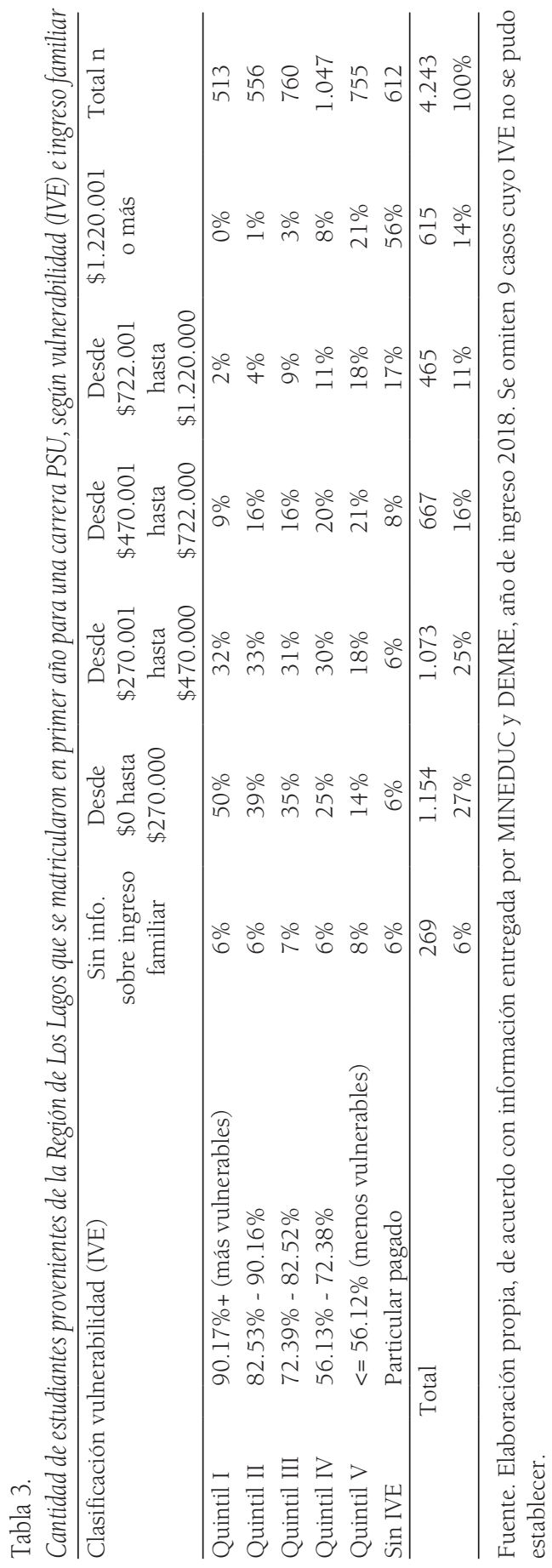


Para la medición del nivel socioeconómico de los estudiantes, la utilización del indicador de IVE del establecimiento secundario presenta ventajas por sobre la variable ingresos, por razones de pragmatismo y eficacia. Por un lado, la información disponible entrega los ingresos en bandas que no es posible comparar entre las dos bases de datos — la de PSU y la de gratuidad—. Además, los datos de ingresos en las bases PSU y gratuidad corresponden en gran medida a datos autoinformados. En cambio, la cantidad de estudiantes en programas sociales que se utiliza para el cálculo de IVE corresponde a datos administrativos. Por otro lado, el IVE y el nivel educacional de los padres refleja en gran medida la situación socioeconómica de los estudiantes, sin necesidad de recurrir a los ingresos.

\subsection{Análisis de los datos}

Específicamente, la metodología abarca dos ámbitos de análisis de los datos indicados: a) una comparación de las características del grupo de estudiantes, provenientes de la Región de Los Lagos, que ingresaron el 2018 a primer año a carreras universitarias que utilizan la PSU como método de selección, y del subgrupo de ellos que se adjudicaron gratuidad. Para esta comparación se abordan las variables de origen de los estudiantes y del destino de sus estudios universitarios. b) Un análisis del tipo de institución universitaria y de su localización geográfica donde se matricula el grupo y subgrupo de estudiantes antes detallado. Esto con el fin de describir la permanencia o traslado por estudios universitarios.

En cuanto a los procedimientos cuantitativos para dichos análisis, en primer lugar se elaboran gráficos de distribución. En segundo, respecto de la movilidad territorial, se aplican test estadísticos para estudiar si las relaciones encontradas son significativas.

De acuerdo con lo planteado, las variables relevantes para el estudio corresponden a variables categóricas, cuyo análisis presenta dos restricciones debido a las muestras utilizadas. Primero, las dos muestras no son independientes entre sí, ya que la muestra denominada "gratuidad" es un subconjunto de la muestra que 
62 ACCESO A LA EDUCACIÓN UNIVERSITARIA Y GRATUIDAD: MOVILIDAD EDUCACIONAL Y MOVILIDAD TERRITORIAL EN UNA REGIÓN NO METROPOLITANA DE CHILE - C. Pareja Pineda, O. Mac-Clure, C. Pérez Vásquez

denominamos "universo PSU". Por esta razón, no es posible realizar pruebas estadísticas para comparar esas muestras en cuanto al nivel de la influencia relativa de variables específicas. Segundo, si bien estas muestras son censales para la cohorte de ingreso del año estudiado, en el marco del análisis de relaciones entre las variables se considera que ambas son muestras dentro de la población conformada por todas las cohortes de ingreso a lo largo de los años.

En cuanto a las herramientas estadísticas inferenciales que se utilizan, éstas abarcan dos tipos de test. El primer test relevante corresponde al de significancia del coeficiente de correlación $\chi^{2}$. Este permite establecer si la hipótesis nula de independencia entre las variables es posible de rechazar o no (Agresti, 2002; Bryman, 2012). Si bien este test permite establecer la posible existencia de una relación, no permite cuantificarla. Para estos fines utilizamos el coeficiente $V$ de Kramer. Este indicador varía entre 0 y 1 , donde a mayor valor se estaría en presencia de una relación más importante (Bryman, 2012). Dicho coeficiente permite revisar si la relación es más fuerte en el universo PSU o en el subgrupo "gratuidad". Sin embargo, esta medición es más bien descriptiva, pues no es posible realizar un test que concluya si la diferencia entre los indicadores es estadísticamente significativa, al estar comparando muestras no independientes.

El segundo test relevante utilizado se aplica cuando las variables categóricas corresponden a datos ordinales, en cuyo caso el test analiza la existencia de una relación monotónica entre las dos variables estudiadas. Este test se basa en el coeficiente gamma de Goodman y Kruskal. Sobre la base del supuesto de una relación monotónica, es posible calcular aquellos casos en que se cumple que, a mayor valor de una categoría, el valor de la otra sube (o baja); a partir de este cálculo se construye el coeficiente (Agresti, 2002). El coeficiente gamma varía entre - 1 y 1 y se interpreta de manera similar al coeficiente correlación $\mathrm{R}^{2}$, de modo que, a mayor valor absoluto del coeficiente, mayor es la relación. El signo positivo indica si la relación es creciente y, por el contrario, un signo negativo que la relación es decreciente ${ }^{9}$.

9 Para todos los análisis estadísticos se utilizó el software SPSS. 


\section{Resultados y discusión}

4.1. Caracterización del acceso a la universidad en el universo de estudiantes y con gratuidad, en la Región de Los Lagos

Los estudiantes provenientes de la Región de los Lagos y de establecimientos secundarios tradicionales que se matricularon en primer año en universidades vía PSU durante 2018 fueron 4.252 personas, de un total de 11.853 postulantes provenientes de la región en el mismo año. Entre estos estudiantes de la región, 1.934 obtuvieron gratuidad (un 45\%). Este porcentaje es similar al $42 \%$ del total del país.

En términos de género ${ }^{10}$, quienes adscriben a la gratuidad en la región estudiada son levemente más mujeres. En el universo PSU, las mujeres alcanzan un $55 \%$, lo que se eleva a un 59\% entre quienes reciben gratuidad. Por otro lado, quienes se identifican con una etnia se elevan del $25 \%$ al $36 \%$.

Al estudiar la cantidad de estudiantes matriculados según categoría del IVE, se aprecia que aquellos establecimientos de mayor vulnerabilidad están más representados en la gratuidad que en el universo de estudiantes PSU. Los estudiantes provenientes de establecimientos con mayor IVE, es decir los más vulnerables, alcanzan un $12 \%$ en el universo PSU, mientras que aumentan a un $22 \%$ entre los estudiantes con gratuidad, según se aprecia en la figura 1.

10 Lamentablemente, solo se cuenta con datos en términos binarios hombre/mujer; los autores reconocen la diversidad existente más allá de estas dos categorías. 
64 ACCESO A LA EDUCACIÓN UNIVERSITARIA Y GRATUIDAD: MOVILIDAD EDUCACIONAL Y MOVILIDAD TERRITORIAL EN UNA REGIÓN NO METROPOLITANA DE CHILE - C. Pareja Pineda, O. Mac-Clure, C. Pérez Vásquez

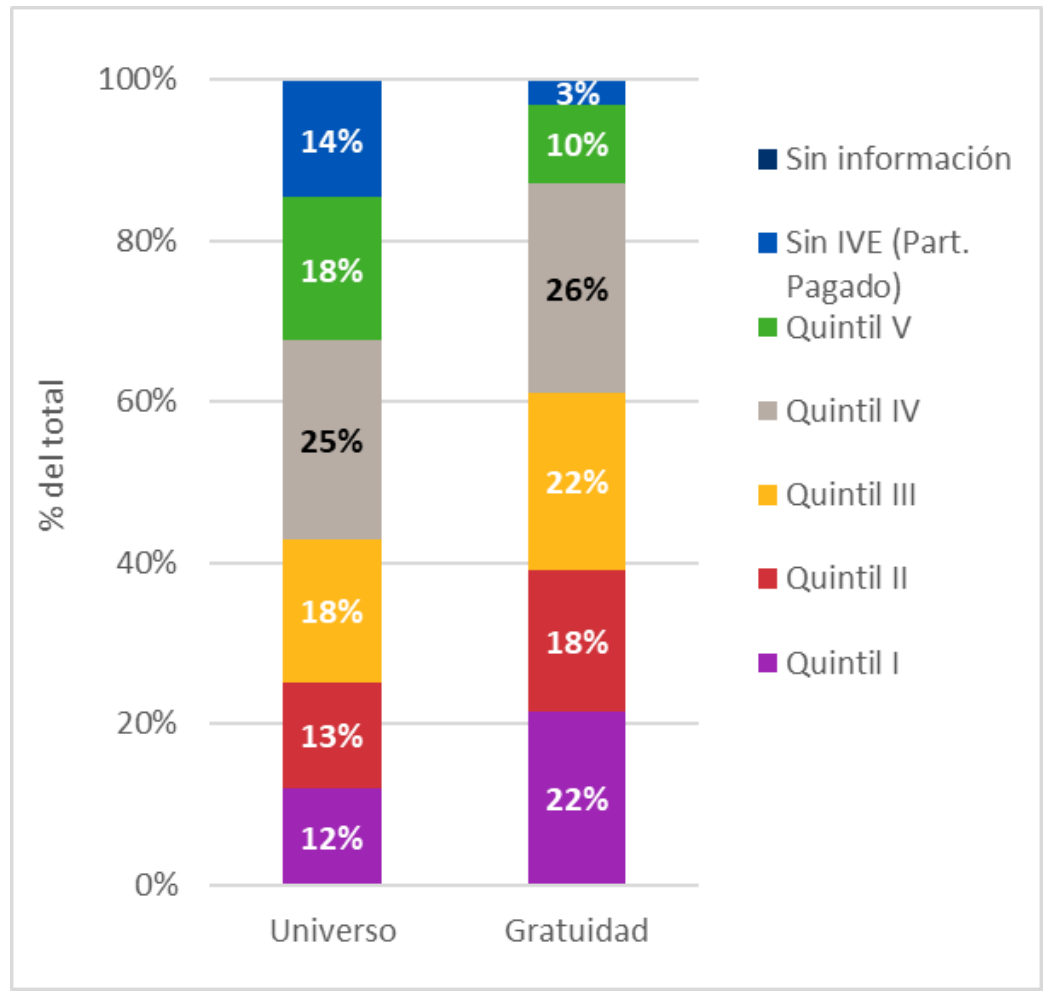

Figura 1. Proporción (\%) de matriculados en primer año en carreras PSU según categoría IVE del establecimiento secundario de egreso.

Región de Los Lagos 2018. N = 1.934 gratuidad; 4.252 universo.

Fuente. Elaboración propia, de acuerdo con información entregada por MINEDUC y DEMRE.

De un modo general, sumando los estudiantes provenientes de establecimientos con una vulnerabilidad del $56 \%$ o más, entre los matriculados PSU estos estudiantes alcanzan a un $68 \%$. En el subconjunto de los que obtuvieron gratuidad representan un $87 \%$ de los matriculados. Considerando este índice de vulnerabilidad como indicador de nivel socioeconómico, la gratuidad claramente abarca a los estudiantes menos privilegiados.

En un sentido similar, los datos muestran también que los estudiantes provenientes de establecimientos municipales son más representados en el subgrupo "gratuidad", en desmedro de los 
establecimientos particulares pagados. La figura 2 muestra cómo los establecimientos municipales pasan desde un 36\% a un $48 \%$, los particulares pagados de un 15\% a un 3\%, mientras que los particulares subvencionados mantienen su participación prácticamente sin cambio.

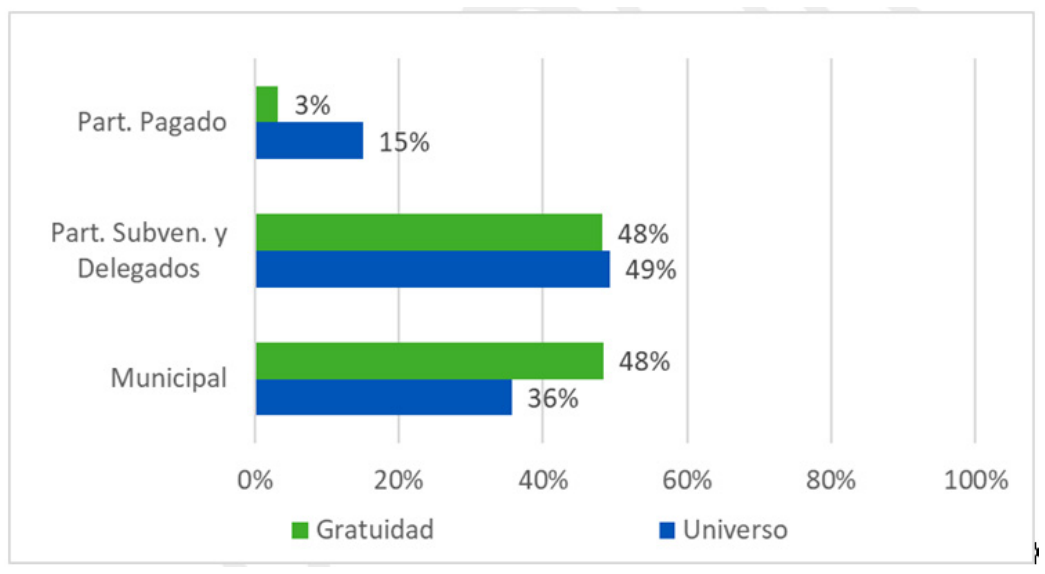

Figura 2. Proporción (\%) de matriculados en primer año en carreras PSU según dependencia establecimiento secundario de egreso.

Región de Los Lagos 2018. N = 1.934 gratuidad; 4.252 universo.

Fuente. Elaboración propia, de acuerdo con información entregada por MINEDUC y DEMRE.

La proporción de matriculados según nivel educativo máximo del padre y/o la madre, distinguiendo entre el conjunto de los matriculados PSU y los con gratuidad, se aprecia en la figura 3. Se observa que un 39\% de los matriculados PSU proviene de hogares donde el padre y/o la madre cursaron estudios universitarios, proporción que disminuye a $17 \%$ entre estudiantes con gratuidad. Así, descontando las personas para quienes no existe información, al menos un $70 \%$ de los estudiantes con gratuidad constituyen la primera generación con estudios universitarios en su familia. De este modo, la gratuidad estaría siendo focalizada en aquellos estudiantes cuyo acceso a la educación universitaria sería más crucial, con miras a una movilidad educacional intergeneracional. 
66 ACCESO A LA EDUCACIÓN UNIVERSITARIA Y GRATUIDAD: MOVILIDAD EDUCACIONAL Y MOVILIDAD TERRITORIAL EN UNA REGIÓN NO METROPOLITANA DE CHILE - C. Pareja Pineda, O. Mac-Clure, C. Pérez Vásquez

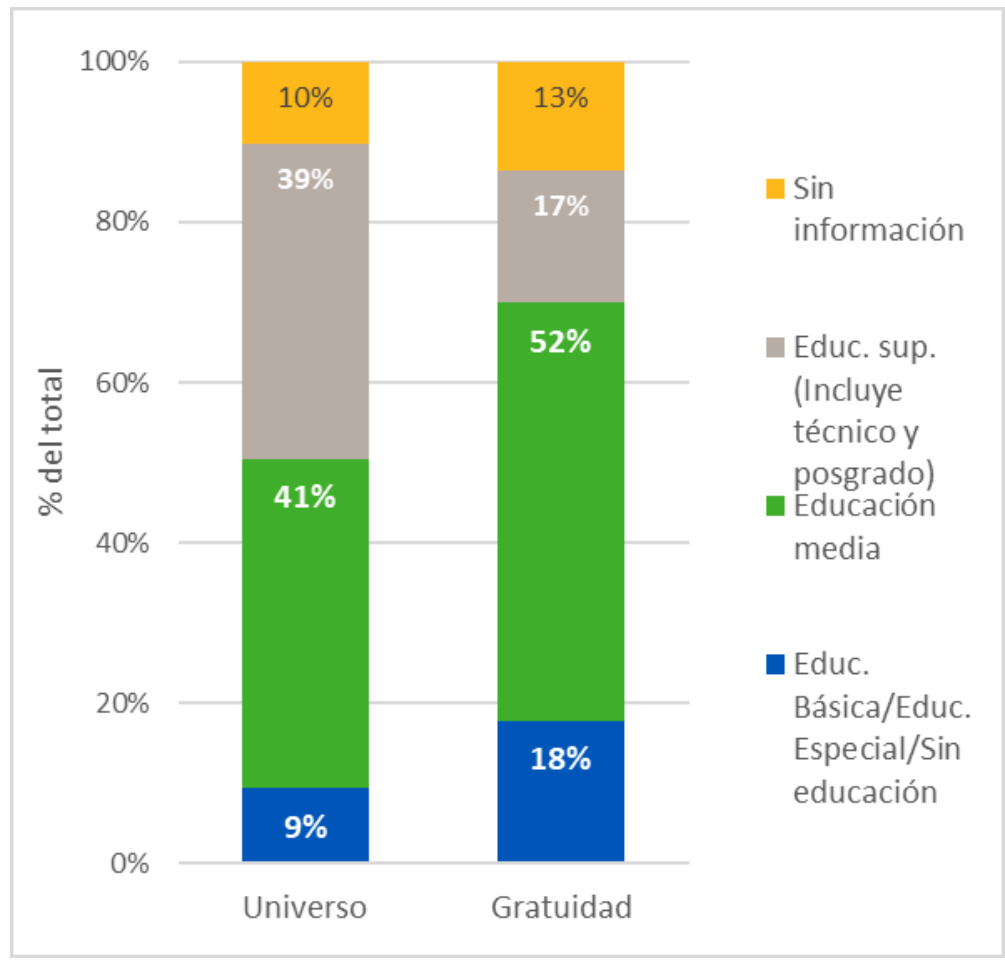

Figura 3. Proporción (\%) de matriculados en primer año en carreras PSU según nivel educativo máximo del padre y/o la madre.

Región de Los Lagos 2018. N = 1.934 gratuidad; 4.252 universo.

Fuente. Elaboración propia, de acuerdo con información entregada por MINEDUC y DEMRE.

Al analizar el tipo de institución universitaria donde se matriculan los estudiantes con ingreso PSU, se observa una marcada prevalencia de instituciones privadas $\mathrm{CRUCH}$, en las cuales se matricula un $44,4 \%$ de los estudiantes, seguidas por las universidades estatales y las privadas, con un $28,3 \%$ y $27,3 \%$ de la matrícula respectivamente. En comparación, en el subgrupo de estudiantes con gratuidad aumenta a un 53\% la matrícula en universidades privadas CRUCH y a un $44 \%$ en estatales, reduciéndose a solo un $3 \%$ la participación de las universidades privadas. Así, en la práctica, las universidades privadas fuera del CRUCH no son una opción para los estudiantes con gratuidad de la región analizada. 
Al incorporar la variable de movilidad, se observa que la mayor matrícula en universidades estatales por parte de estudiantes con gratuidad, en comparación con el universo PSU, se vincula principalmente con estudiantes que no salen de su comuna de origen y con estudiantes que no salen de la región, según se aprecia en la figura 4. La única universidad estatal de la región a la que pueden ingresar estudiantes con gratuidad es la Universidad de Los Lagos. En esta universidad estatal se matricularon 817 estudiantes con gratuidad, de un total de 2.119 estudiantes matriculados en las universidades de la región. La proporción de estudiantes que no sale de su región de origen y que se matricula en universidades estatales, varía desde un $41 \%$ en el universo PSU a un $68 \%$ entre estudiantes con gratuidad. De esta manera, se observa que la oferta universitaria regional cumple un rol crucial respecto de la gratuidad.

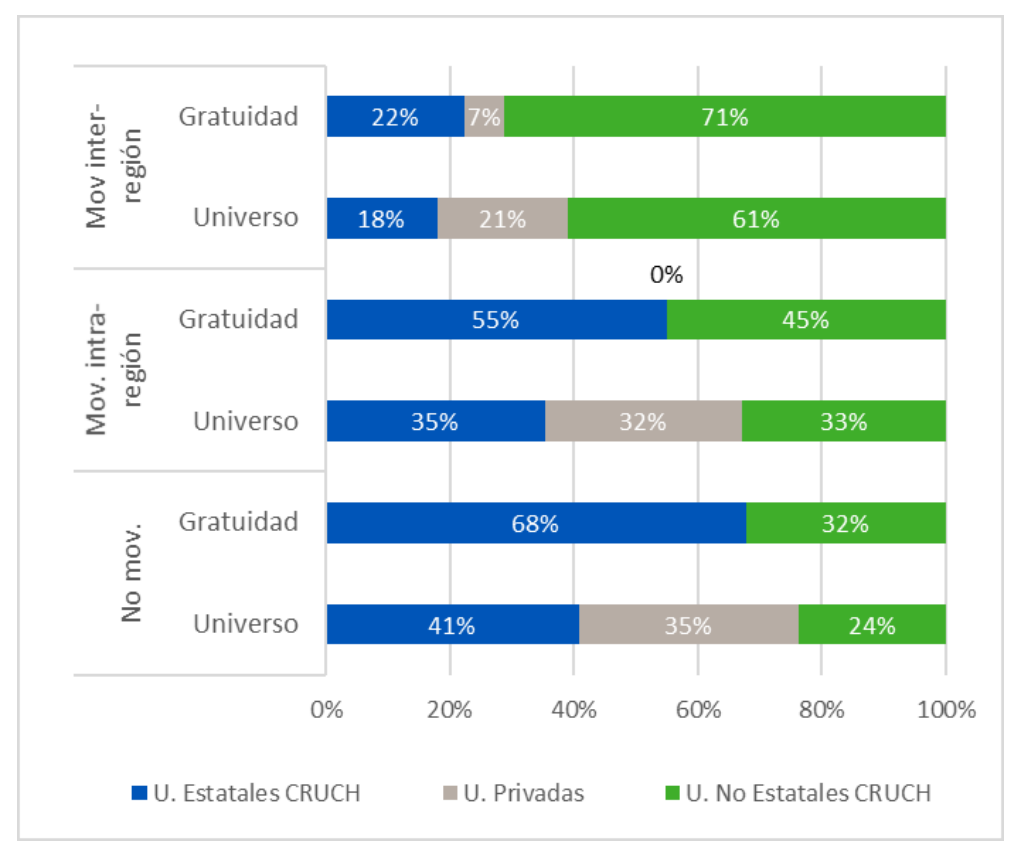

Figura 4. Proporción de estudiantes de primer año en carreras PSU por tipo de institución donde se matricula y según tipo de movilidad.

"No movilidad" indica el estudiante se quedó en su territorio (e.g. comuna) de origen. Región de Los Lagos 2018. N = 1.934 gratuidad; 4.252 universo.

Fuente. Elaboración propia, de acuerdo con información entregada por MINEDUC y DEMRE. 
68 ACCESO A LA EDUCACIÓN UNIVERSITARIA Y GRATUIDAD: MOVILIDAD EDUCACIONAL Y MOVILIDAD TERRITORIAL EN UNA REGIÓN NO METROPOLITANA DE CHILE - C. Pareja Pineda, O. Mac-Clure, C. Pérez Vásquez

4.2. Relación entre las características sociales de origen y la movilidad territorial para acceder a la universidad

Según lo expuesto, resulta particularmente relevante analizar en qué medida quienes permanecen dentro de la región para acceder a la universidad son principalmente estudiantes de menor estatus socioeconómico, mientras que los que se desplazan entre regiones son principalmente estudiantes de mayor estatus. Si se confirma que este es el patrón, examinaremos si es modificado por la gratuidad y de qué manera ocurriría eso. Respecto de estas preguntas, además de describir lo que indican los datos, la aplicación de técnicas estadísticas inferenciales permitirá verificar si existen asociaciones significativas.

La movilidad territorial según destino, sea dentro o fuera de la Región de Los Lagos, así como un detalle de la movilidad intrarregional, se muestra en la figura 5. El panel de la izquierda indica el universo PSU y el de la derecha el subconjunto de los estudiantes con gratuidad. Para identificar la relación entre vulnerabilidad y destino geográfico, se considera el IVE del establecimiento secundario. El porcentaje de quienes estudian en su territorio de origen se indica en la parte superior de cada barra. Se aprecia que los estudiantes provenientes de establecimientos más vulnerables permanecen en la Región de Los Lagos, mientras que el resto tiende a estudiar en otras regiones.

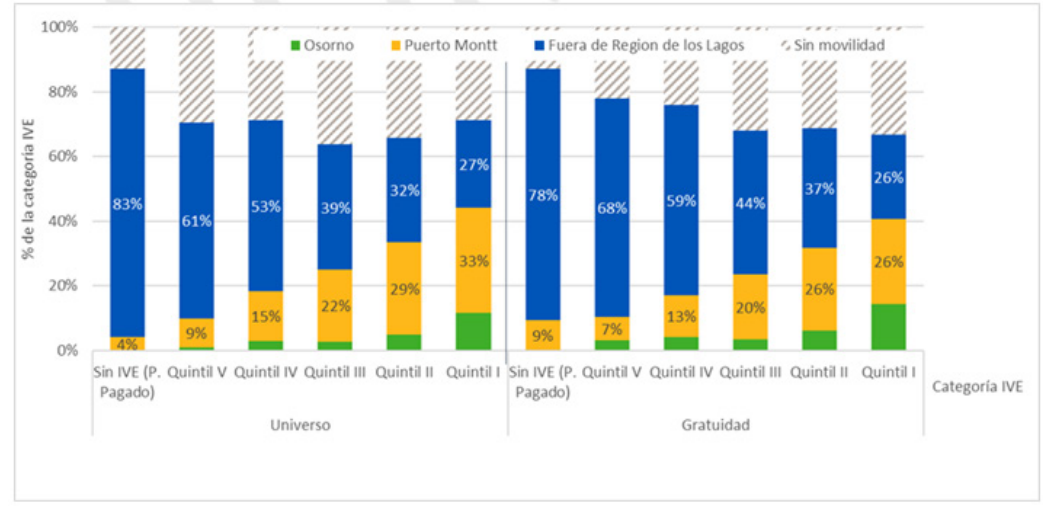

Figura 5. Proporción de estudiantes matriculados en primer año en carreras PSU dentro y fuera de la Región de Los Lagos, según categoría IVE.

Región de los Lagos 2018. N = 1.934 gratuidad (5 casos omitidos por falta de datos); 4.252 universo (sin IVE, 9 casos omitidos).

Fuente: Elaboración propia, de acuerdo con información entregada por MINEDUC y DEMRE. 
En la figura destaca, en primer lugar, que si bien a menor vulnerabilidad es mayor la cantidad de personas que permanece en su territorio de origen, esta pauta es similar tanto en el universo como en el subgrupo "gratuidad". Un segundo fenómeno observable es que hay diferencias intrarregionales entre quienes se movilizan fuera de su territorio de origen, pero se mantienen en la región de la cual son originarios. Al interior de la región, la preferencia es por trasladarse a Puerto Montt más que a Osorno. Más aún, quienes se trasladan a Puerto Montt son mayoritariamente estudiantes provenientes de establecimientos más vulnerables —los tres niveles de mayor IVE- - Un tercer fenómeno que muestra la figura es una relación entre salir de la región y provenir de un establecimiento con menor vulnerabilidad. Esto se observa en el decrecimiento de la proporción de estudiantes que se desplazan fuera de la región de origen al aumentar la vulnerabilidad del establecimiento en el que cursaron estudios secundarios. En cuarto lugar, la figura 5 también muestra que las tres situaciones recién mostradas suceden tanto en el universo estudiado como en el subgrupo "gratuidad". En efecto, es posible ver en la figura que los gráficos del lado izquierdo y derecho no presentan mayor diferencia.

Asumiendo estas cuatro aseveraciones como hipótesis a ser testeadas estadísticamente en forma sucesiva, los resultados se presentan en la tabla 4. En cada fila se informa primero el resultado de la prueba Chi-cuadrada para independencia y luego el estadístico gamma, en caso de que las categorías se encuentren ordenadas, o el indicador $\mathrm{V}$ de Cramer, en caso de que no guarden una ordenación. 
70 ACCESO A LA EDUCACIÓN UNIVERSITARIA Y GRATUIDAD: MOVILIDAD EDUCACIONAL Y MOVILIDAD TERRITORIAL EN UNA REGIÓN NO METROPOLITANA DE CHILE - C. Pareja Pineda, O. Mac-Clure, C. Pérez Vásquez

Tabla 4.

Resultados de las pruebas estadísticas en el universo PSU estudiado y en el subgrupo "gratuidad"

\begin{tabular}{|c|c|c|}
\hline Hipótesis estudiada & Universo & Gratuidad \\
\hline $\begin{array}{l}\text { 1. Relación decreciente } \\
\text { entre presentar movilidad } \\
\text { (no movilidad, movilidad) y } \\
\text { vulnerabilidad (según IVE) }\end{array}$ & $\begin{array}{l}\chi^{2}=104,540 \text { p-valor }=0,000 \\
\text { gamma }=-0,163 \pm 0,043 \\
\text { p-valor }=0,000\end{array}$ & $\begin{array}{l}\chi^{2}=23,561 \mathrm{p} \text {-valor }=0,000 \\
\text { gamma }=-0,155 \pm 0,068 \\
\text { p-valor }=0,000\end{array}$ \\
\hline $\begin{array}{l}\text { 2. Relación entre destino } \\
\text { intrarregional (Osorno o Puerto } \\
\text { Montt) y vulnerabilidad según } \\
\text { IVE }\end{array}$ & $\begin{array}{l}\chi^{2}=28.669 \text { p-valor }=0,000 \\
\text { V de Cramer }=0,179\end{array}$ & $\begin{array}{l}\chi^{2}=18,586 \text { p-valor }=0,000 \\
\text { V de Cramer }=0,195\end{array}$ \\
\hline $\begin{array}{l}\text { 3. Relación decreciente } \\
\text { entre presentar movilidad } \\
\text { interregional (no movilidad } \\
\text { inter., movilidad inter.) y } \\
\text { vulnerabilidad (según IVE) }\end{array}$ & $\begin{array}{l}\chi^{2}=518,724 \text { p-valor }=0,000 \\
\text { gamma }=-0,461 \pm 0,035 \\
\text { p-valor }=0,000\end{array}$ & $\begin{array}{l}\chi^{2}=169,069 \\
\text { p-valor }=0,000 \\
\text { gamma }=-0,411 \pm 0,055 \\
\text { p-valor }=0,000\end{array}$ \\
\hline $\begin{array}{l}\text { 4. Relación creciente entre } \\
\text { presentar movilidad más } \\
\text { larga (regiones ordenadas } \\
\text { según distancia geográfica) y } \\
\text { vulnerabilidad (según IVE) }\end{array}$ & $\begin{array}{l}\chi^{2}=1038,680 \\
\text { p-valor }=0,000 \\
\text { gamma }=-0,444 \pm 0,029 \\
\text { p-valor }=0,000\end{array}$ & $\begin{array}{l}\chi^{2}=264,528 \\
\text { p-valor }=0,000 \\
\text { gamma }=-0,378 \pm 0,047 \\
\text { p-valor }=0,000\end{array}$ \\
\hline
\end{tabular}

Fuente. Elaboración propia, de acuerdo con información entregada por MINEDUC y DEMRE.

Al observar los estadísticos de la primera fila de la tabla, es posible concluir que, si bien no se descarta una relación entre vulnerabilidad y movilizarse, dicha relación, según muestran los indicadores gamma pequeños, es más bien marginal. En la segunda fila de la tabla es posible descartar la independencia entre el destino y la vulnerabilidad del establecimiento, sugiriendo una relación entre elegir Puerto Montt y presentar mayor IVE. De manera similar, la tercera fila de la tabla muestra, a través de un alto valor absoluto para gamma y un signo negativo, que a menor vulnerabilidad es más probable movilizarse fuera de la región. El indicador gamma para la relación monotónica entre movilidad interregional y vulnerabilidad para el universo es de $-0,461 \pm 0,035$ frente a un valor $-0,411 \pm 0,055$ para el subgrupo con gratuidad. En general, los estadísticos refrendan lo apreciado en la figura 5, con la salvedad de que, debido a que la muestra de gratuidad es un subgrupo, no existen test estadísticos para establecer si las diferencias entre el universo y este subconjunto son estadísticamente significativas. 
Focalizándonos en la movilidad interregional, nuestros datos muestran que el universo PSU estudia más en la Región Metropolitana que el subgrupo de estudiantes con gratuidad: un 11,4\% frente a un $6,5 \%$, a la izquierda y la derecha de la figura 6 , respectivamente. Esta situación se da a la inversa en cuanto a estudiar en la Región de Los Lagos (parte superior de la barra), donde el universo estudiado se matricula en un $49,8 \%$ frente a un $54,0 \%$ del subgrupo adscrito a gratuidad.

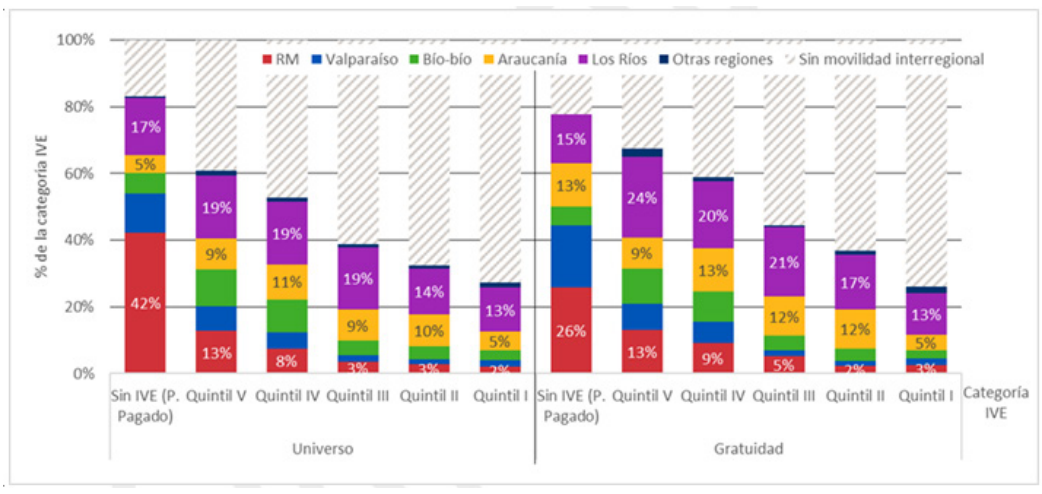

Figura 6. Proporción de estudiantes matriculados en primer año en carreras PSU fuera de Los Lagos, por región destino de la matricula universitaria y según categoría IVE.

Región de los Lagos 2018. N = 1.934 gratuidad (sin IVE, 5 casos omitidos); 4.252 universo (sin IVE, 9 casos omitidos).

Fuente: Elaboración propia, de acuerdo con información entregada por MINEDUC y DEMRE.

Se aprecia en la figura 6 que, desde los establecimientos privados, un $26 \%$ de los estudiantes con gratuidad estudia en Santiago, mientras que solo un 3\% de los estudiantes con gratuidad y de establecimientos más vulnerables (categoría IVE 90\%+) viaja a Santiago. Para quienes estudian fuera de la región y provienen de establecimientos vulnerables, la Región de los Ríos, la más cercana a la Región de Los Lagos, se vuelve el destino predominante. En comparación con el universo de estudiantes, esa región es un destino más frecuente entre los estudiantes con gratuidad, quienes se matriculan en la Universidad Austral, la única adscrita a ese sistema en la Región de Los Ríos. Estudiar en regiones como Araucanía, Biobío y Valparaíso constituye una alternativa para estudiantes de 
72 ACCESO A LA EDUCACIÓN UNIVERSITARIA Y GRATUIDAD: MOVILIDAD EDUCACIONAL Y MOVILIDAD TERRITORIAL EN UNA REGIÓN NO METROPOLITANA DE CHILE - C. Pareja Pineda, O. Mac-Clure, C. Pérez Vásquez

nivel socioeconómico medio, en mayor medida que migrar a Santiago o a la vecina Región de Los Ríos.

Los resultados anteriores sugieren la hipótesis de que para los estudiantes de la Región de Los Lagos existe una relación decreciente entre la vulnerabilidad de su establecimiento de educación secundaria y qué tan distante se movilizan para sus estudios universitarios. Para analizar estadísticamente esta relación decreciente —a mayor vulnerabilidad, movilidad territorial menos distante- se utiliza una variable ordinal de las regiones, que permite evaluar el estadístico gamma y así avanzar más allá del test de independencia. Partiendo de la base de que las regiones están naturalmente ordenadas geográficamente, se asumen dos premisas para el análisis. Primero, se considera conjuntamente a las regiones Metropolitana y Valparaíso, en el entendido de que ambas regiones suponen el mismo esfuerzo migratorio. Segundo, se estudian solo las principales regiones de destino: Metropolitana en conjunto con Valparaíso, Los Ríos, Biobío y Araucanía. Las regiones consideradas abarcan más del 98\% de cada muestra. Con estas premisas se encontró que la relación descrita es estadísticamente significativa, alcanzando un valor gamma de $-0,444 \pm 0,029$ en el universo PSU y de $-0,378 \pm 0,047$ en el subgrupo "gratuidad", según se presenta en la cuarta fila de la tabla 4. Es decir, existiría una relación de mediana magnitud entre una menor vulnerabilidad y una mayor movilidad territorial para matricularse en una región geográficamente más distante. Sin embargo, pareciera que el vínculo según vulnerabilidad del establecimiento es más fuerte en el universo del conjunto de estudiantes, pues el valor absoluto de gamma es mayor en comparación al valor encontrado para el subgrupo con gratuidad. Lo anterior indicaría que, entre quienes acceden a gratuidad, desplazarse más lejos no dependería tan fuertemente de la vulnerabilidad del establecimiento de origen, sugiriendo otras variables condicionantes para este subgrupo.

En línea con lo anterior, el género también parece influir en la movilidad territorial para acceder a la universidad, según se aprecia en la figura 7. 


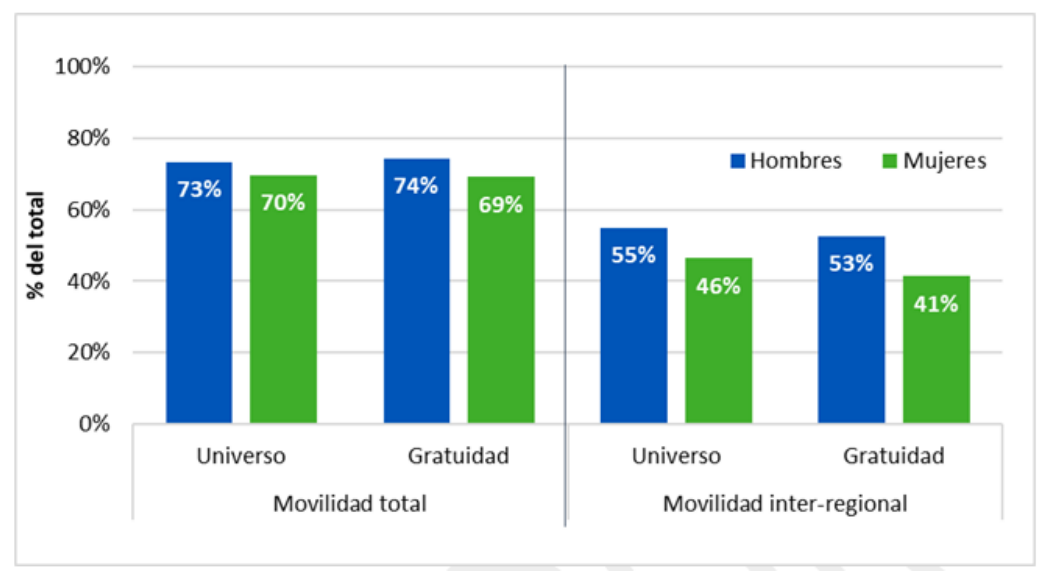

Figura 7. Proporción de los matriculados en primer año en carreras PSU que se moviliza, según género

El panel de la izquierda muestra la movilidad total (intra e interregional) y el de la derecha la interregional. Región de Los Lagos 2018. N = 1.934 gratuidad; 4.252 universo.

Fuente: Elaboración propia, de acuerdo con información entregada por MINEDUC y DEMRE.

Si bien las mujeres son quienes más entran a las universidades y quienes más frecuentemente obtienen la gratuidad, los resultados indican que son los hombres quienes más se desplazan, como sugiere la figura 7. Estas diferencias entre hombres y mujeres aumentan levemente en el subgrupo de estudiantes adscritos a la gratuidad - como se aprecia gráficamente en el costado derecho de la figura-.

Estas asociaciones enunciadas entre género y movilidad territorial son analizadas como hipótesis estadísticamente verificables en la tabla 5.

Tabla 5.

Resultados de las pruebas estadísticas relacionadas a la posible asociación entre género y movilidad

\begin{tabular}{lll}
\hline \multicolumn{1}{c}{ Hipótesis } & \multicolumn{1}{c}{ Universo } & \multicolumn{1}{c}{ Gratuidad } \\
\hline $\begin{array}{lll}\text { 1. Independencia entre presentar } \\
\text { movilidad o no y género }\end{array}$ & $\chi^{2}=6,726$ p-valor $=0,010$ & $\chi^{2}=6,363$ p-valor $=0,012$ \\
\hline $\begin{array}{ll}\text { 2. Independencia entre presentar } \\
\text { movilidad interregional o no y género V de Cramer }=0,085\end{array}$ & $\chi^{2}=31,054$-valor $=0,000$ & $\chi^{2}=23,227$ p-valor $=0,000$ \\
\hline
\end{tabular}

Fuente: Elaboración propia, de acuerdo con información entregada por MINEDUC y DEMRE. 
74 ACCESO A LA EDUCACIÓN UNIVERSITARIA Y GRATUIDAD: MOVILIDAD EDUCACIONAL Y MOVILIDAD TERRITORIAL EN UNA REGIÓN NO METROPOLITANA DE CHILE - C. Pareja Pineda, O. Mac-Clure, C. Pérez Vásquez

La diferencia de género al comparar la movilidad total (intra e interregional) es pequeña y no estadísticamente significativa, según muestra la primera fila de la tabla 5. Sin embargo, esta desigualdad de género se acentúa en la movilidad interregional, la cual es estadísticamente significativa, como es posible observar en la segunda fila de la tabla, es decir, los hombres desarrollarían mayor movilidad interregional. Más aún, esto se verifica numéricamente al comparar los índices V de Cramer en la misma fila de la tabla, los cuales indican que este fenómeno se acentúa en el subgrupo "gratuidad". En efecto, en el universo el indicador alcanza un valor de 0,085 y, si bien el alza es pequeña, en el subgrupo alcanza un 0,110 .

Los resultados presentados confirman estadísticamente que los estudiantes de menor estatus socioeconómico acceden a universidades de la región donde residen, según se aprecia en el caso de la Región de Los Lagos. Existe un patrón de movilidad en el que estudiantes hombres socialmente más aventajados presentan mayor movilidad territorial que los y las demás. El universo PSU y el subgrupo "gratuidad" muestran un mismo patrón en cuanto a que, a menor vulnerabilidad mayor movilidad, pero difieren en el destino de los estudios: los estudiantes con gratuidad suelen tener movilidades más cercanas que el resto.

\section{Conclusiones}

Los resultados presentados en este estudio muestran que, en el caso de la Región de Los Lagos, la gratuidad está focalizada en aquellos grupos que tradicionalmente han tenido menos oportunidades en el acceso a la educación universitaria, favoreciendo la movilidad educacional. La gratuidad abarca especialmente a los estudiantes provenientes de establecimientos con mayor vulnerabilidad escolar y de establecimientos municipales.

Al mismo tiempo, el estudio proporciona evidencia de que la movilidad territorial de los estudiantes, entendida como estudiar fuera del territorio o de la región de origen, está estrechamente vinculada al acceso a la educación universitaria, en el caso de una región no metropolitana como la estudiada. Sin embargo, la movilidad 
territorial constituye un fenómeno que varía según las características sociales y territoriales de los estudiantes. Quienes se desplazan entre regiones son principalmente estudiantes de mayor estatus socioeconómico; más aún, a mayor estatus, mayor preferencia por Santiago. Los grupos menos privilegiados permanecen en la región para realizar sus estudios universitarios, aunque con un alto nivel de movilidad territorial al interior de ella. Asimismo, la universidad estatal presente en la región se vuelve clave para estudiantes que acceden a la gratuidad.

Lo anterior supone dos problemas. Primero, respecto de la movilidad interregional, si a estudiantes con gratuidad se les facilitaran condiciones similares a sus pares más privilegiados, esto supondría asumir los costos financieros que conlleva migrar fuera de su región de origen, junto con establecer los apoyos no monetarios necesarios para que dicha migración sea exitosa; una alternativa consiste en mejorar y ampliar la oferta universitaria regional con los costos que eso también conlleva. Segundo, la alta movilidad intrarregional presente en una región como Los Lagos puede suponer una dificultad en el desempeño académico de estudiantes con gratuidad, aspecto actualmente no cubierto en este mecanismo de financiamiento.

Nuestros hallazgos indican que las universidades regionales, específicamente la Universidad de Los Lagos y la Universidad Austral, son más demandadas entre estudiantes con gratuidad que en el universo de ingresados a la universidad por PSU. Esto hace que el sistema universitario público en una región como la de Los Lagos sea clave en las posibilidades educacionales de los estudiantes menos privilegiados. De esta manera, la permanencia de los estudiantes adscritos a la gratuidad en la región de origen es a la vez una oportunidad para las regiones, pero también un posible "cuello de botella" para las oportunidades de formación universitaria, haciéndose clave la calidad y variedad de la oferta actual.

En efecto, la insuficiencia de los recursos aportados a las universidades estatales regionales en el marco de la política de gratuidad (Brunner y Labraña, 2018, pp. 17-19; Sanhueza, 2019), dificulta una mejor oferta a los estudiantes de la región, a pesar de 
76 ACCESO A LA EDUCACIÓN UNIVERSITARIA Y GRATUIDAD: MOVILIDAD EDUCACIONAL Y MOVILIDAD TERRITORIAL EN UNA REGIÓN NO METROPOLITANA DE CHILE - C. Pareja Pineda, O. Mac-Clure, C. Pérez Vásquez

los avances logrados en la calidad de la educación universitaria. En las universidades estatales regionales los recursos aportados por la política de gratuidad son insuficientes para un mejoramiento de prácticas docentes y de la calidad de la docencia universitaria, para el desarrollo de investigación pedagógica sobre esos temas y de la investigación científica para favorecer un medio académico universitario integral, y, en general, para el fortalecimiento de la oferta de estas universidades en beneficio de los estudiantes que más lo necesitan, por sus condiciones socioeconómicas. Por lo tanto, para que un subsidio a la demanda como la gratuidad sea eficaz requiere ser parte de una política más amplia, que incluya un mejoramiento de la disponibilidad y calidad de la oferta educacional de las universidades estatales regionales. Es importante notar que, en Chile, el subsidio a la demanda en la educación escolar ha profundizado la segregación educativa (Villalobos y Quaresma, 2015), fenómeno que debe evitarse se trasmita a la educación universitaria a través de la gratuidad.

En síntesis, según hemos descrito, la gratuidad tiene efectivamente el potencial de ampliar el acceso a la universidad de estudiantes de condiciones socioeconómicas menos privilegiadas, lo cual abre la puerta para que, tras la graduación, con su carrera puedan tener la oportunidad de un proceso de movilidad social y, además, hacerlo sin el peso de una deuda por sus estudios. La gratuidad conlleva un mensaje claro de que puede estudiar en la universidad quien tiene un origen socioeconómico y territorial menos favorable, lo que se ha logrado efectivamente, de acuerdo con los resultados empíricos de este estudio.

En estudios posteriores, sería de interés examinar otros factores en juego en el caso de los estudiantes con gratuidad, como sus disposiciones subjetivas o su rol en el grupo familiar, que podrían afectar su decisión acerca de dónde estudiar. Asimismo, es importante estudiar el efecto de la trayectoria de educación secundaria sobre la permanencia o retención en la universidad y la titulación oportuna, especialmente en el caso de los estudiantes con gratuidad. También será relevante evaluar el impacto de la gratuidad en cuanto a la inserción laboral de los egresados universitarios, para concluir si 
la movilidad educacional efectivamente se traduce en movilidad ocupacional y social.

Sin embargo, aunque los resultados de esta investigación muestran que la gratuidad estaría bien focalizada, en el sentido de que quienes se adscriben a ella son los menos privilegiados, por sí sola ella no resuelve un problema de inequidad educacional más amplia, anterior al ingreso a la universidad. A esto se agrega enseguida el carácter segregador de las pruebas de ingreso, las dificultades académicas al ingresar a la universidad por parte de los estudiantes menos privilegiados, la aguda estratificación de los centros de estudio universitarios y la insuficiencia de los recursos transferidos a las universidades estatales regionales. Este escenario se agrava en el caso de los estudiantes provenientes de los espacios territoriales no metropolitanos del país. En definitiva, la gratuidad, por sí sola, no tiene la capacidad de remover completamente barreras importantes que limitan las perspectivas de un acceso equitativo a la educación universitaria, particularmente a nivel territorial.

\section{Referencias}

Abarca, G., y Zapata, C. (2005). Indígenas y educación superior: El caso mapuche. Calidad en la Educación, (26), 57-80. DOI: http://dx.doi. org/10.31619/caledu.n26.233

Agresti, A. (2002). Categorical Data Analysis (Second Edition). Nueva York: John Wiley \& Sons.

Álvarez Rivadulla, M. J. (2019). ¿"Los becados con los becados y los ricos con los ricos"? Interacciones entre clases sociales distintas en una universidad de elite. Desacatos. Revista De Ciencias Sociales, (59), 50. DOI: $10.29340 / 59.2049$

Angrist, J., Autor, D., Hudson, S. \& Pallais, A. (2016). Evaluating postsecondary aid: Enrollment, persistence, and projected completion effects. DOI: https://doi.org/10.3386/w23015

Barozet, E., Contreras, D., Espinoza, V., Gayo, M. y Méndez, M. L. (2021). Clases medias en tiempos de crisis: vulnerabilidad persistente, desafíos para la cohesión y un nuevo pacto social en Chile. Santiago de Chile: CEPAL.

Bourdieu, P. (2007). Efectos de lugar. En La miseria del mundo. Buenos Aires: Fondo de Cultura Económica. 
78 ACCESO A LA EDUCACIÓN UNIVERSITARIA Y GRATUIDAD: MOVILIDAD EDUCACIONAL Y MOVILIDAD TERRITORIAL EN UNA REGIÓN NO METROPOLITANA DE CHILE - C. Pareja Pineda, O. Mac-Clure, C. Pérez Vásquez

Bravo, M. y Verdugo, S. (2007). Gestión escolar y éxito académico en condiciones de pobreza. REICE. Revista Iberoamericana sobre Calidad, Eficacia y Cambio en Educación, 5(1). Recuperado el 30 de diciembre de 2019, de https://www.redalyc.org/articulo.oa?id=55100107

Brunner, JJ. y Labraña, J. (2018). Financiamiento de la educación superior, gratuidad y proyecto de nuevo crédito estudiantil. Centro de Estudios Públicos (CEP), Debates de Políticas Públicas, (31), 1-26. Recuperado el 30 de septiembre de 2019, de https://www.cepchile.cl/cep/site/ docs/20181115/20181115124945/dpp_031_noviembre2018_ jjbrunner_jlabrana.pdf

Bryman, A. (2012). Social research methods (Fourth edition). Oxford University Press.

Bucarey, A. (2018). Who pays for free college? Crowding out on campus. Job market paper. MIT Department of Economics.

Bucarey, A., Contreras, D. \& Muñoz, P. (2020). Labor Market Returns to Student Loans for University: Evidence from Chile. Journal of Labor Economics, 38(4), 959-1007.

Canales, A. (2016). Diferencias socioeconómicas en la postulación a las universidades chilenas: El rol de factores académicos. Calidad en la educación, (44), 129-157. DOI: https://doi.org/10.4067/S071845652016000100006

Carvalhaes, F. \& Ribeiro, C. A. C. (2019). Estratificação horizontal da educação superior no Brasil: Desigualdades de classe, gênero e raça em um contexto de expansão educacional. Tempo Social, 31(1), 195 233. DOI: https://doi.org/10.11606/0103-2070.ts.2019.135035

Castillo, J. y Cabezas, G. (2010). Caracterización de jóvenes de primera generación en educación superior, nuevas trayectorias hacia la equidad educativa. Calidad en la Educación, (32), 44-76. DOI: https://doi. org/10.31619/caledu.n32.151

Castillo, P., González, A. y Puga, I. (2011). Gestión y efectividad en educación: Evidencias comparativas entre establecimientos municipales y particulares subvencionados. Estudios Pedagógicos, 37(1), 187-206. DOI: https://doi.org/10.4067/S0718-07052011000100010

Delisle, J. \& Bernasconi, A. (2018). Lessons from Chile's transition to free college. Evidence Speaks, 2(43), 14. Recuperado el 30 de enero de 2020, de https://www.brookings.edu/wp-content/uploads/2018/03/ delisle-and-bernasconi-report.pdf

Donoso, S., Arias, O., Weason, M. y Frites, C. (2012). La oferta de educación superior de pregrado en chile desde la perspectiva territorial: 
Inequidades y asimetrías en el mercado. Calidad de la educación, (37), 100-127. DOI: https://doi.org/10.4067/S0718-45652012000200004

Espinoza, Ó., González-Fiegehen, L.-E. y Granda, M. (2019). Avances y desafíos que enfrentan los procesos reformistas en la educación superior de Chile y Ecuador: La perspectiva sobre el acceso y el financiamiento. Revista Iberoamericana de Educación Superior, X(27), 25-55. DOI: https://doi.org/dx.doi.org/10.22201/iisue.20072872e.2019.27.339

Espinoza, Ó., y González, L. E. (2013). Access to higher education in Chile: A public vs. private analysis. Prospects, 43(2), 199-214. DOI: https:// doi.org/10.1007/s11125-013-9268-8

Espinoza, Ó. y González, L. E. (2015). Equidad en el sistema de educación superior de Chile: Acceso, permanencia, desempeño y resultados. En A. Bernasconi (Ed.), La educación superior de Chile: Transformación, desarrollo y crisis (p. 632). Santiago de Chile: Universidad Católica de Chile.

Espinoza, V. y Núñez, J. (2014). Movilidad ocupacional en Chile 2001-2009: ¿Desigualdad de ingresos con igualdad de oportunidades? Revista Internacional de Sociologia, 72(1), 57-82. DOI: https://doi.org/10.3989/ ris.2011.11.08

Filho Crepalde, N. J. B. \& Silvera Souza, L. (2016). Desempenho universitário no Brasil: Estudo sobre desigualdade educacional com dados do ENADE 2014. Revista Brasileira de Sociologia, 4(7), 211-240. DOI: https://doi.org/10.20336/rbs. 155

Goldrick-Rab, S., Kelchen, R., Harris, D. N. \& Benson, J. (2016). Reducing income inequality in educational attainment: Experimental evidence on the impact of financial aid on college completion. American Journal of Sociology, 121(6), 1762-1817. DOI: https://doi.org/10.1086/685442

González, P., Arancibia, V. y Boyanova, D. (2017). Talento académico, vulnerabilidad escolar y resultados en la prueba de selección universitaria. Estudios Pedagógicos, 43(1), 171-191. DOI: https://doi. org/10.4067/S0718-07052017000100011

Hidalgo, R., De Mattos, C. y Arenas, F. (2009). Del país urbano al país metropolitano. Transformaciones recientes en las ciudades chilenas. Santiago de Chile: Colección Eure-Libros.

Jarpa-Arriagada, C. G. y Rodríguez-Garcés, C. (2018). Segmentación y exclusión en Chile: El caso de los jóvenes primera generación en educación superior. Revista Latinoamericana de Ciencias Sociales, Niñez y Juventud, (15), 327-343. DOI: https://doi.org/10.11600/169271 $5 x .1512028032016$ 
80 ACCESO A LA EDUCACIÓN UNIVERSITARIA Y GRATUIDAD: MOVILIDAD EDUCACIONAL Y MOVILIDAD TERRITORIAL EN UNA REGIÓN NO METROPOLITANA DE CHILE - C. Pareja Pineda, O. Mac-Clure, C. Pérez Vásquez

López, I., Mella, J. y Cáceres, G. (2018). La universidad como ruptura en la trayectoria educativa: Experiencias de transición de estudiantes egresados de enseñanza media técnico profesional que ingresan al Programa Académico de Bachillerato de la Universidad de Chile. Estudios Pedagógicos, 44(3), 291-308. Recuperado el 30 de septiembre de 2019, de http://revistas.uach.cl/index.php/estped/article/ view/4233/5283

Loveday, V. (2014). Working-class participation, middle-class aspiration? Value, upward mobility and symbolic indebtedness in higher education. The Sociological Review, 63(3), 570-588.

Marteleto, L., Marschner, M. \& Carvalhaes, F. (2016). Educational stratification after a decade of reforms on higher education access in Brazil. Research in Social Stratification and Mobility, (46), 99-111. DOI: https://doi. org/10.1016/j.rssm.2016.08.004

Ministerio de Educación MINEDUC. (s. f.). ¿Cómo funciona el Sinae? - JUNAEB. Recuperado el 29 de agosto de 2019, de https://www.junaeb.cl/comofunciona-el-sinae

Muñoz, M. y Blanco, C. (2013). Una taxonomía de las universidades chilenas. Calidad en la Educación, (38), 181-213. DOI: http://dx.doi. org/10.31619/caledu.n38.109

OECD. (2018). A broken social elevator? How to promote social mobility. OECD Publishing. https://doi.org/10.1787/9789264301085-en

Reay, D. (2018). Working class educational transitions to university: The limits of success. European Journal of Education, 53(4), 528-540. DOI: https://doi.org/10.1111/ejed.12298

Riquelme, H. (2017). Cruzando la Araucanía (Chile). Un análisis de las prácticas de movilidad cotidiana de universitarios adscritos a la Gratuidad. Cuhso, 27(1), 23-50. DOI: https://doi.org/10.7770/ CUHSO-V27N1-AR

Ruff Escobar, C., Ruiz Toledo, M., Matheu Pérez, A. y Juica Martínez, P. (2020). Análisis de las políticas de financiamiento mixto en educación superior y sus efectos en la movilidad social y en la investigación, el caso de Chile. Gestión y Política Pública, 29(2), 413. DOI: 10.29265/ gypp.v29i2.779

Sabatini, F., Cáceres, G. y Cerda, J. (2001). Segregación residencial en las principales ciudades chilenas: Tendencias de las tres últimas décadas y posibles cursos de acción. Eure, 27(82), 21-42. DOI: https://doi. org/10.4067/s0250-71612001008200002 
Sanhueza, P. (2019). Universidades perjudicadas por apoyar la gratuidad. El Mercurio de Valparaíso, 10. Recuperado el 30 de septiembre de 2019, de http://www.mercuriovalpo.cl/impresa/2019/09/08/full/cuerpoprincipal/10/

Santiago, P., Tremblay, K., Basri, E., \& Arnal, E. (2008). Tertiary education for the knowledge society (Vol. 1). In Tertiary Education for the Knowledge Society (Vol. 1). DOI: https://doi.org/10.1787/9789264063518-hu

Saravia, F. A. (2018a). La dimensión territorial de las desigualdades en trayectorias sociales de profesionales: el caso de la Región de Los Lagos (tesis doctoral). Universidad de Los Lagos.

Saravia, F. A. (2018b). Vínculo entre percepción territorial y movilidad espacial en trayectorias de la clase media profesional: Región de Los Lagos, Chile. Cadernos Metrópole, 20(42), 531-551. DOI: https://doi. org/10.1590/2236-9996.2018-4211

Sepúlveda, L. y Valdebenito, M. J. (2014). ¿Las cosas claras? Aspiraciones de futuro y proyecto educativo laboral de jóvenes estudiantes secundarios. Estudios pedagógicos, 40(1), 243-261. DOI: https://doi.org/10.4067/ S0718-07052014000100015

Solis, A. (2017). Credit access and college enrollment. Journal of Political Economy, 125(2), 562-622. DOI: https://doi.org/10.1086/690829

Torche, F. (2005). Unequal but fluid: Social mobility in Chile in comparative perspective. American Sociological Review, (70), 422-450.

Torche, F. (2014). Movilidad intergeneracional y desigualdad: El caso latinoamericano. Annual Review of Sociology, 40(1), S2-1-S2-25. DOI: https://doi.org/10.1146/annurev-soc-062215-092006

Valdivieso, P., Antivilo, A. y Barrios, J. (2006). Caracterización sociodemográfica de estudiantes que rinden la PSU, postulan y se matriculan en universidades del Consejo de Rectores. Calidad en la Educación, (24), 312. DOI: https://doi.org/10.31619/caledu.n24.281

Villalobos, C. y Quaresma, M. L. (2015). Sistema escolar chileno: Características y consecuencias de un modelo orientado al mercado. Convergencia, 22(69), 63-84. DOI: https://doi.org/10.29101/crcs. v22i69.3634

Recibido: 18/05/2021

Aceptado: 29/09/2021 